\title{
Threshold optimization of a finite sample-based cognitive radio network using energy detector
}

\author{
Ajay Singh ${ }^{1}$, Manav R Bhatnagar ${ }^{2 *}$ and Ranjan K Mallik²
}

\begin{abstract}
In this paper, we consider a cognitive radio network containing two cognitive radios (CRs) and one primary user. The CRs utilize finite number of received data samples for estimating the energy of the primary signals and forward these energy estimates to a fusion center (FC). The FC combines the energy estimates and utilizes a global threshold based on the exact knowledge of local thresholds of the CRs for determining the presence or absence of the primary signal. We propose selective and semi-selective soft combining schemes for this set-up. For the proposed schemes, we derive the total probability of error of detecting a spectrum hole. By minimizing the total probability of error in sensing a spectrum hole, we find optimized local and global thresholds. Moreover, we also discuss the optimization of conventional non-selective soft and 1-bit hard combining schemes with multiple (equal to or more than two) collaborative CRs under the total probability of error minimization criterion. It is shown by simulations that the proposed selective soft combination-based scheme significantly outperforms the conventional non-selective schemes based on soft combination and 1-bit hard combination. Further, it is shown by simulation that the proposed selective soft combining scheme along with the total probability of error minimization criterion is able to properly utilize a spectrum hole with interference level less than the standard specified value.
\end{abstract}

Keywords: Cognitive radio; Collaborative networks; Energy detector; Probability of error; Spectrum hole detection

\section{Introduction}

Due to the utilization of the radio electromagnetic spectrum up to saturation, there is scarcity of free radio spectrum [1,2]. However, it is seen in practice that even the dedicated radio spectrum is not used efficiently. For example, in television broadcasting, the spectrum allocated is idle for considerable time. This free spectrum can be utilized for some other applications using a cognitive radio network [1,2]. Hence, effective utilization of the spectrum plays an important role in today's crowded spectrum environment. Whenever required, a licensed user (primary user) can use its licensed band, otherwise the spectrum can be used for secondary operations. A spectrum hole is said to exist when a particular band

\footnotetext{
*Correspondence: manav@ee.iitd.ac.in

2 Department of Electrical Engineering, Indian Institute of Technology - Delhi,

Hauz Khas, New Delhi 110016, India

Full list of author information is available at the end of the article
}

of frequencies is not used by the primary user. Searching for spectrum holes is termed as spectrum sensing [1]. Sensing of the spectrum holes is done by the cognitive radios (CRs) which are unlicensed users or secondary users [1,2]. A CR is an intelligent wireless communication system that periodically monitors the radio spectrum and detects the occupancy of the different parts of the spectrum. The CR opportunistically communicates over spectrum holes without interfering with the primary user's signals [3].

There are many challenges in detecting a spectrum hole. Few of these are fading, noise uncertainty, and shadowing. These effects may lead to significant interference to a licensed user. It is shown in the literature [4-8] that cooperation among the CRs can be utilized in order to overcome the problems of fading, noise uncertainty, and shadowing. An optimal linear cooperation framework of spectrum sensing for detecting the primary signals is proposed in [4]. The cooperative spectrum sensing in [4] is based on linear combination of local statistics from

\section{是 Springer}

(c) 2013 Singh et al: licensee Springer. This is an Open Access article distributed under the terms of the Creative Commons

Attribution License (http://creativecommons.org/licenses/by/2.0), which permits unrestricted use, distribution, and reproduction in any medium, provided the original work is properly cited. 
individual CRs. In [4], each CR uses an energy detector, which estimates the energy of the primary signal from the received data samples. These energy estimates are forwarded over noiseless channels to a fusion center (FC). The performance of the cognitive system is optimized using Neyman-Pearson (NP) criterion by assuming that large number of received data samples are used by each CR for energy estimation in [4]. In [7], a binary decisionbased cooperative spectrum sensing scheme is discussed for cognitive networks. Each CR takes a binary decision using energy detector about the presence or absence of the primary signal. The binary decisions are forwarded to the FC which combines them for taking final decision. The sum of probability of missed opportunity and probability of false alarm is minimized for obtaining an optimized threshold for the CRs. Since the hard decision is taken at the CRs in [7], the performance of this scheme is poorer than a soft decision-based scheme [9]. In a soft decision-based scheme, the CRs forward the energy estimates to the FC and FC combines them in order to take the decision about the presence of the primary signal. In [8], the set-up of [7] is generalized to the scenario when the CRs collect very large number of data samples to take binary decisions. The optimized value of the local threshold in the CRs is numerically calculated by minimizing the total probability of error.

Energy detector is proposed for cognitive spectrum sensing because it needs no information about the primary signal and has lower complexity in real-time detection of spectrum hole. For the detection of unknown deterministic signals corrupted by the additive white Gaussian noise (AWGN), an energy detector is derived in [10]. Performance analysis of the energy detector for random signals is studied in [11]. The performance of the energy detector for unknown transmit signal in AWGN and fading environment is discussed in [12]. In [13], optimal soft combination scheme for received energy data is explained. The detail study regarding the performance of spectrum sensing in different scenario is done in [14-24].

In this paper, we consider a collaborative cognitive radio network which utilizes a finite number of samples for deciding the presence or absence of the primary signals. The cognitive network contains two secondary users and one FC. We consider a cognitive set-up, where the CRs do not take decision about the spectrum hole based on their local thresholds. Nevertheless, they can update the local thresholds as per the channel between the primary user and the CRs. These local thresholds are conveyed to the FC and the FC updates its global threshold accordingly. The CRs forward their energy estimates to the FC over noiseless reporting channels and the FC takes decision about the spectrum hole based on the normalized global and local thresholds. In selective soft combining, the FC combines the energy estimates of both CRs for making a decision of the primary signal only if their energy estimates are above the local threshold.

Expressions of the probability of false alarm and the probability of missed opportunity for this collaborative system are derived for the proposed selective combing scheme. The global and local thresholds are optimized by minimizing the total probability of error in detection of a spectrum hole. The proposed scheme of selective soft combination significantly outperforms the conventional soft and 1-bit hard combining schemes. It is also shown that the total probability of error minimization criterion is able to utilize a spectrum hole more efficiently as compared to the NP criterion while keeping the interference to the primary user $(\mathrm{PU})$ within the desired limits for the signal-to-noise ratio (SNR) of the PU-CR link considered in simulations.

The rest of the paper is organized as follows. In Section 2, the system model of the cognitive radio network is explained. Performance analysis of the proposed scheme of selective soft combining and semi-selective soft combining is discussed in Section 3. Optimization of the normalized global and local thresholds is also performed in this section.

Section 4 discusses optimization of non-selective soft combining and 1-bit hard combining schemes for arbitrary number of CRs under the total probability of error minimization criterion. Numerical results are discussed in Section 5. In Section 6, some conclusions are drawn.

\section{System model}

We consider a cognitive network consisting of two secondary users $\mathrm{S}_{1}$ and $\mathrm{S}_{2}$, one primary user and a fusion center FC, as shown in Figure 1. In this cognitive radio network, it is possible that the spectrum allocated for the PU is not utilized at some time; therefore, the secondary users monitor the spectrum continuously in order to make sure that detection of signals transmitted by the PU cannot be ignored at any instance of time. To enhance the reliability of spectrum usage, interference to the PU from secondary users should be kept very low [3]. In our analysis, we will

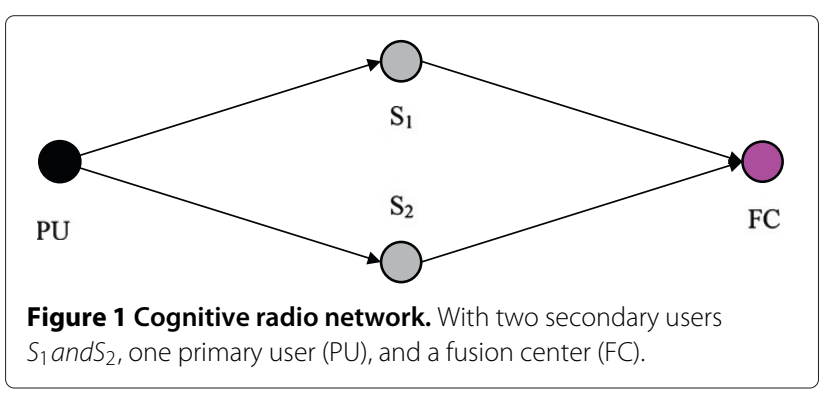


focus on how to minimize the interference to the PU while minimizing the probability of error in detecting the spectrum hole such that the free spectrum can be efficiently utilized. It is assumed that only one PU is in operation with highest priority to use its allocated spectrum as compared to each secondary user. In the cognitive network considered in Figure 1, there exists two hypotheses at the $k$-th time instant

$$
\begin{array}{lll}
H_{0}: & y_{i}(k)=v_{i}(k), & \text { if } \mathrm{PU} \text { is absent, } \\
H_{1}: & y_{i}(k)=s h_{i}(k)+v_{i}(k), & \text { if } \mathrm{PU} \text { is present, }
\end{array}
$$

where $y_{i}$ is the received signal in $i$-th CR, $i$ is the secondary user index $(i=1,2)$, $s$ denotes the signal transmitted by the primary user with energy $E_{s}, v_{i}(k) \sim \mathcal{C N}\left(0, \sigma_{n}^{2}\right)$ is circularly symmetrical complex additive white Gaussian noise (AWGN), and $h_{i}(k) \sim \mathcal{C N}\left(0, \sigma_{h}^{2}\right)$ is the complex normal circularly symmetrical channel gain between the PU and $i$-th CR, implying Rayleigh fading. The variance of the signal received at each secondary user under hypothesis $H_{0}$ is $\sigma_{n}^{2}$, while that under $H_{1}$ is given by

$$
\sigma_{h n}^{2}=\sigma_{h}^{2} E_{s}+\sigma_{n}^{2} .
$$

We assume that the local threshold of detection for each secondary user is the same and is given as $\lambda$. This assumption is valid when both CRs exist at the same distance from the PU. CRs can update $\lambda$ according to the SNR ratio of the links between the PU and the CRs. It is assumed that the value of $\lambda$ is also known in the FC. We call $\lambda$ as local threshold because of local spectrum sensing is performed at individual CR. As decision is taken globally in the FC, we refer $\lambda_{0}$ by global threshold of detection in the FC. Energy estimates of each CR normalized with number of samples is termed as summary statistics in this paper. Each secondary user calculates a summary statistics $u_{i}$ over a finite detection interval of $L$ samples as

$$
u_{i}=\frac{1}{L} \sum_{k=0}^{L-1}\left|y_{i}(k)\right|^{2} .
$$

The summary statistics are transmitted to the FC through a lossless control channel using orthogonal time slots or orthogonal frequency bands. The FC computes a global test statistics as

$$
U=u_{1}+u_{2}
$$

The characteristic function (c.f.) of $U$ obtained from Eq. (3) in the case of hypothesis $H_{0}$ is given as [25]

$$
\psi_{U \mid H_{0}}(j \omega)=\frac{1}{\left(1-\frac{j \omega \sigma_{n}^{2}}{L}\right)^{2 L}},
$$

Similarly, from Eq. (3), the c.f. of $U$ under hypothesis $H_{1}$ will be [25]

$$
\psi_{U \mid H_{1}}(j \omega)=\frac{1}{\left(1-\frac{j \omega \sigma_{h n}^{2}}{L}\right)^{2 L}} .
$$

By taking the inverse Fourier transform of Eqs. (5) and (6), the probability density functions (p.d.f.s) of $U$ under hypotheses $H_{0}$ and $H_{1}$ can be obtained as

$$
f_{U \mid H_{0}}(x)=\frac{L^{2 L} \exp \left(-\frac{x L}{\sigma_{n}^{2}}\right) x^{2 L-1}}{\sigma_{n}^{4 L} \Gamma(2 L)}
$$

and

$$
f_{U \mid H_{1}}(x)=\frac{L^{2 L} \exp \left(-\frac{x L}{\sigma_{h n}^{2}}\right) x^{2 L-1}}{\sigma_{h n}^{4 L} \Gamma(2 L)},
$$

respectively, where $\Gamma(a)$ is the Gamma function [26, Eq. (6.1.1)].

\section{Performance analysis of the combining schemes}

In binary hypothesis problem, we are interested in finding the hypothesis which is true. It may be $H_{1}$ or $H_{0}$ depending upon the decision region. Here, we will focus our attention to the Bayes criterion for decision based on the received data. The expression of the Bayesian risk $R$ can be written using [27, Eq. (5), Chapter (2)] as follows:

$$
\begin{aligned}
R= & C_{00} P_{0} \int_{Z_{0}} f_{U \mid H_{0}}(x) d x+C_{10} P_{0} \int_{Z_{1}} f_{U \mid H_{0}}(x) d x \\
& +C_{11} P_{1} \int_{Z_{1}} f_{U \mid H_{1}}(x) d x+C_{01} P_{1} \int_{Z_{0}} f_{U \mid H_{1}}(x) d x
\end{aligned}
$$

where $P_{0}$ and $P_{1}$ denote a priori probabilities of hypotheses $H_{0}$ and $H_{1}$, respectively, $Z_{1}$ and $Z_{0}$ are the decision regions corresponding to hypotheses $H_{1}$ and $H_{0}$, respectively, while $C_{00}, C_{10}, C_{11}, C_{01}$ denote cost assigned as follows [27]:

1. $C_{00}$ corresponds to the cost of deciding hypothesis $H_{0}$ when hypothesis $H_{0}$ is true,

2. $C_{10}$ corresponds to the cost of deciding hypothesis $H_{1}$ when hypothesis $H_{0}$ is true,

3. $C_{11}$ corresponds to the cost of deciding hypothesis $H_{1}$ when hypothesis $H_{1}$ is true,

4. $C_{01}$ corresponds to the cost of deciding hypothesis $H_{0}$ when hypothesis $H_{1}$ is true.

It is assumed that $C_{00}=C_{11}=0$ which implies that no cost is assigned when correct hypothesis is chosen. This assumption is valid because no error is made if the decision of the presence or absence of the primary signal is correct. However, in order to reduce the probability of error in decision, we have to set $C_{01}=$ $C_{10}=1$ so that maximum cost is assigned for a wrong decision. 
In $\mathrm{CR}$ spectrum sensing, when the primary signal is present and the $C R$ decides that it is not present, then it causes interference to the PU and leads to overutilization of the spectrum. Similarly, when the CR decides a the PU signal is present and actually it is not, then spectrum hole is underutilized. Therefore, we consider that cost assigned in both types of error is set as unity.

For designing a minimum total probability of errorbased receiver, we also assume that two hypotheses $H_{0}$ and $H_{1}$ are equally likely [27] such that $P_{0}=P_{1}=0.5$. With these assumptions, Eq. (9) can be written as

$$
R=\frac{1}{2} \int_{Z_{1}} f_{U \mid H_{0}}(x) d x+\frac{1}{2} \int_{Z_{0}} f_{U \mid H_{1}}(x) d x .
$$

It can be deduced that Eq. (10) refers to the total probability of error or average probability of error in deciding the presence or absence of the primary signal. In our analysis, we have chosen this type of error criterion specifically in order to minimize the total probability error. It will be shown in Section 5 that the total probability of error criterion enables the cognitive system to utilize free spectrum more efficiently than the NP criterion, for certain SNR values considered in simulations.

The probability of false alarm $P_{f}$ and probability of missed detection $P_{m}$ are defined as [27]

$$
\begin{aligned}
& P_{f} \triangleq \int_{Z_{1}} f_{U \mid H_{0}}(x) d x, \\
& P_{m} \triangleq \int_{Z_{0}} f_{U \mid H_{1}}(x) d x .
\end{aligned}
$$

Therefore, substituting Eqs. (11) and (12) in Eq. (10), we get

$$
R=\frac{1}{2} P_{f}+\frac{1}{2} P_{m}
$$

For the two-CR-based cognitive set-up considered in Section 2 , decision regions $Z_{1}$ corresponding to the detection of the PU are given as

1. $u_{1}>\lambda, u_{2}>\lambda$, and $u_{1}+u_{2}>\lambda_{0}$,

2. $u_{1}>\lambda, u_{2}<\lambda$, and $u_{1}+u_{2}>\lambda_{0}$,

3. $u_{1}<\lambda, u_{2}>\lambda$, and $u_{1}+u_{2}>\lambda_{0}$,

4. $u_{1}<\lambda, u_{2}<\lambda$, and $u_{1}+u_{2}>\lambda_{0}$.

The decision regions corresponding to non-detection of the PU, i.e., $Z_{0}$ will be complimentary to the decision regions of $Z_{1}$ in all above conditions. The detection region corresponding to $u_{1}<\lambda, u_{2}<\lambda$, and $u_{1}+u_{2}>\lambda_{0}$ is non-zero for $\lambda_{0}<2 \lambda$ only.

\subsection{Proposed selective combining scheme}

It is beneficial to check the received signal at each CR and include those signals, which are above a local threshold $\lambda$, in making the final decision in the FC. Let us consider a soft combining-based scheme, where the FC takes decision of the presence or absence of the spectrum hole when $u_{1}>\lambda, u_{2}>\lambda$, and $u_{1}+u_{2}>$ $2 \lambda$. Hence, selective combining in the FC concerns with the fact that both CRs contribute in the decision about the presence or absence of the primary signal provided that energy estimates of both CRs are above the local threshold $\lambda$. Intuitively, the selective combining scheme guarantees the minimum total probability of error in the detection of spectrum hole. Motivated by this fact, we consider a proposed scheme for soft combination of received data in which decision region is $u_{1}>\lambda, u_{2}>$ $\lambda$, and $u_{1}+u_{2}>\lambda_{0}$. We will evaluate the performance of the proposed selective combining scheme by deriving the expressions of the probability of false alarm and the probability of missed opportunity for this collaborative system.

\subsection{Total probability of error of the selective combining scheme}

For finding performance analysis of the proposed soft combining scheme, we split our analysis into three parts $\lambda_{0}>2 \lambda, \lambda_{0}=2 \lambda$, and $\lambda_{0}<2 \lambda$.

\subsubsection{Total probability of error of the selective combining scheme for $\lambda_{0}>2 \lambda$}

From Figure 2, it is found that the detection region for primary signal is shaded region labeled as $Z_{1}$. The probability of false alarm $P_{f_{1}}$ can be obtained from Eq. (11) and Figure 2 as follows:

$$
\begin{aligned}
P_{f_{1}}= & \mathcal{I}\left(\lambda, \infty, \lambda, \infty, \sigma_{n}^{2}, L, x, y\right) \\
& -\mathcal{I}\left(\lambda, \lambda_{0}-\lambda, \lambda, \lambda_{0}-x, \sigma_{n}^{2}, L, x, y\right),
\end{aligned}
$$

where

$$
\begin{aligned}
\mathcal{I}\left(a, b, c, d, \sigma_{n}^{2}, L, x, y\right) \triangleq & \int_{a}^{b} \int_{c}^{d L^{2 L} \exp \left(-\frac{x L}{\sigma_{n}^{2}}\right) x^{2 L-1}} \\
& \times \frac{L_{n}^{4 L} \Gamma(2 L)}{L^{2 L} \exp \left(-\frac{y L}{\sigma_{n}^{2}}\right) y^{2 L-1}} d y d x .
\end{aligned}
$$

Further simplifying Eq. (14), we get

$$
\begin{aligned}
P_{f_{1}}= & \frac{1}{[\Gamma(2 L)]^{2}} \int_{\frac{L \lambda}{\sigma_{n}^{2}}}^{\frac{L\left(\lambda_{0}-\lambda\right)}{\sigma_{n}^{2}}} \Gamma\left(2 L, \frac{L \lambda_{0}}{\sigma_{n}^{2}}-t\right) t^{2 L-1} \exp (-t) d t \\
& +\frac{1}{[\Gamma(2 L)]^{2}}\left[\Gamma\left(2 L, \frac{L \lambda}{\sigma_{n}^{2}}\right)\right]^{2} \\
& -\frac{1}{[\Gamma(2 L)]^{2}} \Gamma\left(2 L, \frac{L \lambda}{\sigma_{n}^{2}}\right) \Gamma\left(2 L, \frac{L \lambda}{\sigma_{n}^{2}}\right) \\
& +\frac{1}{[\Gamma(2 L)]^{2}} \Gamma\left(2 L, \frac{L \lambda}{\sigma_{n}^{2}}\right) \Gamma\left(2 L, \frac{L\left(\lambda_{0}-\lambda\right)}{\sigma_{n}^{2}}\right),
\end{aligned}
$$




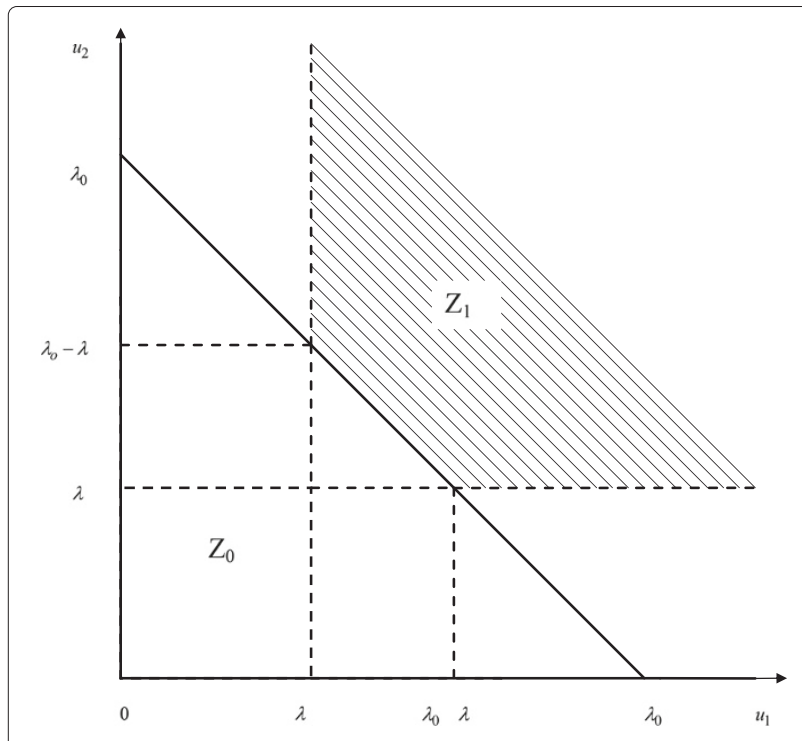

Figure 2 Decision regions for detection and non-detection of the primary signal for $\lambda_{0}>\mathbf{2} \lambda$. Shaded region $Z_{1}$ corresponds to the detection region of the primary signal and unshaded region corresponds to $Z_{0}$ which denotes the region of non-detection of the primary signal.
After simplification of Eq. (18), we get

$$
\begin{aligned}
P_{m_{1}}= & 1-\frac{1}{\Gamma(2 L)} \exp \left(-\frac{L \lambda_{0 n}}{1+\gamma}\right) \sum_{l=0}^{2 L-1} \sum_{i=0}^{l}\left(\begin{array}{l}
l \\
i
\end{array}\right) \\
& \times \frac{(-1)^{l-i}\left(\frac{L \lambda_{0 n}}{1+\gamma}\right)^{i}\left[\left(\frac{L \lambda_{0 n}-L \lambda_{n}}{1+\gamma}\right)^{l+2 L-i}-\left(\frac{L \lambda_{n}}{1+\gamma}\right)^{l+2 L-i}\right]}{l !(l+2 L-i)} \\
& -\frac{1}{[\Gamma(2 L)]^{2}} \Gamma\left(2 L, \frac{L \lambda_{n}}{1+\gamma}\right) \Gamma\left(2 L, \frac{L \lambda_{0 n}-L \lambda_{n}}{1+\gamma}\right),
\end{aligned}
$$

where $\gamma=E_{s} \sigma_{h}^{2} / \sigma_{n}^{2}$ is the average signal-to-noise ratio (SNR) of the PU-CR link.

Total probability of error for $\lambda_{0}>2 \lambda$ can be obtained using Eq. (13) as

$$
P_{e_{1}}=\frac{P_{f_{1}}+P_{m_{1}}}{2} \text {. }
$$

\subsubsection{Total probability of error of the selective combining scheme for $\lambda_{0}=2 \lambda$}

The expression of probability of false alarm $P_{f_{2}}$ for the proposed scheme can be obtained using Eq. (11) and Figure 3 as

$$
P_{f_{2}}=\mathcal{I}\left(\lambda, \infty, \lambda, \infty, \sigma_{n}^{2}, L, x, y\right)=\frac{1}{[\Gamma(2 L)]^{2}}\left[\Gamma\left(2 L, L \lambda_{n}\right)\right]^{2} .
$$

$\Gamma(a, x)=(a-1) ! e^{-x} \sum_{k=0}^{a-1} \frac{x^{k}}{k !}$ in Eq. (16) and after some algebraic simplification of the integral, we have

$$
\begin{aligned}
P_{f_{1}}= & \frac{1}{\Gamma(2 L)} \exp \left(-L \lambda_{0 n}\right) \sum_{l=0}^{2 L-1} \sum_{i=0}^{l}\left(\begin{array}{l}
l \\
i
\end{array}\right) \\
& \times \frac{(-1)^{l-i}\left(L \lambda_{0 n}\right)^{i}\left[\left(L \lambda_{0 n}-L \lambda_{n}\right)^{l+2 L-i}-\left(L \lambda_{n}\right)^{l+2 L-i}\right]}{l !(l+2 L-i)} \\
& +\frac{1}{[\Gamma(2 L)]^{2}} \Gamma\left(2 L, L \lambda_{n}\right) \Gamma\left(2 L, L \lambda_{0 n}-L \lambda_{n}\right)
\end{aligned}
$$

where $\lambda_{0 n}=\lambda_{0} / \sigma_{n}^{2}$ denotes the normalized global threshold, $\lambda_{n}=\lambda / \sigma_{n}^{2}$ denotes the normalized local threshold, and $\left(\begin{array}{l}l \\ i\end{array}\right)=\frac{l !}{i !(l-i) !}$. The expression of probability of missed detection $P_{m_{1}}$ for the proposed selective combining scheme can be obtained using Eq. (12) and Figure 2 as

$$
\begin{aligned}
P_{m_{1}}= & \mathcal{I}\left(0, \infty, 0, \infty, \sigma_{h n}^{2}, L, x, y\right)-\mathcal{I}\left(\lambda, \infty, \lambda, \infty, \sigma_{h n}^{2}, L, x, y\right) \\
& +\mathcal{I}\left(\lambda, \lambda_{0}-\lambda, \lambda, \lambda_{0}-x, \sigma_{h n}^{2}, L, x, y\right)
\end{aligned}
$$

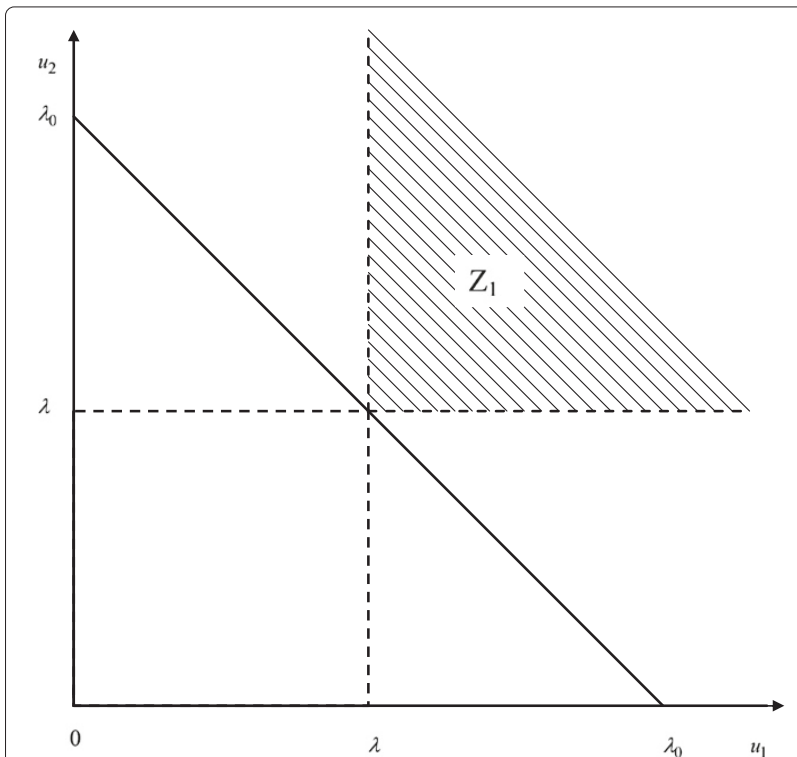

Figure 3 Decision regions for detection and non-detection of the primary signal for $\lambda_{\mathbf{0}}=\mathbf{2} \boldsymbol{\lambda}$. Shaded region $Z_{1}$ corresponds to the detection region of the primary signal and unshaded region corresponds to $Z_{0}$ which denotes the region of non-detection of the primary signal. 
From Figure 3 and Eq. (12), the probability of missed detection $P_{m_{2}}$ of the proposed scheme will be

$P_{m_{2}}=\mathcal{I}\left(0, \infty, 0, \infty, \sigma_{h n}^{2}, L, x, y\right)-\mathcal{I}\left(\lambda, \infty, \lambda, \infty, \sigma_{h n}^{2}, L, x, y\right)$,

that reduces into

$$
P_{m 2}=1-\frac{1}{[\Gamma(2 L)]^{2}}\left[\Gamma\left(2 L, \frac{L \lambda_{n}}{1+\gamma}\right)\right]^{2} .
$$

We can obtain the expression of the total probability of error for $\lambda_{0}=2 \lambda$ using Eq. (13) as

$$
P_{e_{2}}=\frac{P_{f_{2}}+P_{m_{2}}}{2}
$$

\subsubsection{Total probability of error for proposed scheme when}

$$
\lambda_{0}<2 \lambda
$$

It can be seen from Figures 3 and 4 that the detection region of the primary signal $Z_{1}$ for $\lambda_{0}<2 \lambda$ is the same as that of $\lambda_{0}=2 \lambda$; therefore, the probability of false alarm $P_{f_{3}}$ for $\lambda_{0}<2 \lambda$ is given by Eq. (21). Similarly, it can be observed from Figures 3 and 4 that the probability of missed detection for $\lambda_{0}<2 \lambda$ will be given using Eq. (23). Hence, the total probability of error $P_{e_{3}}$ of $\lambda_{0}<2 \lambda$ is equal to $P_{e_{2}}$.

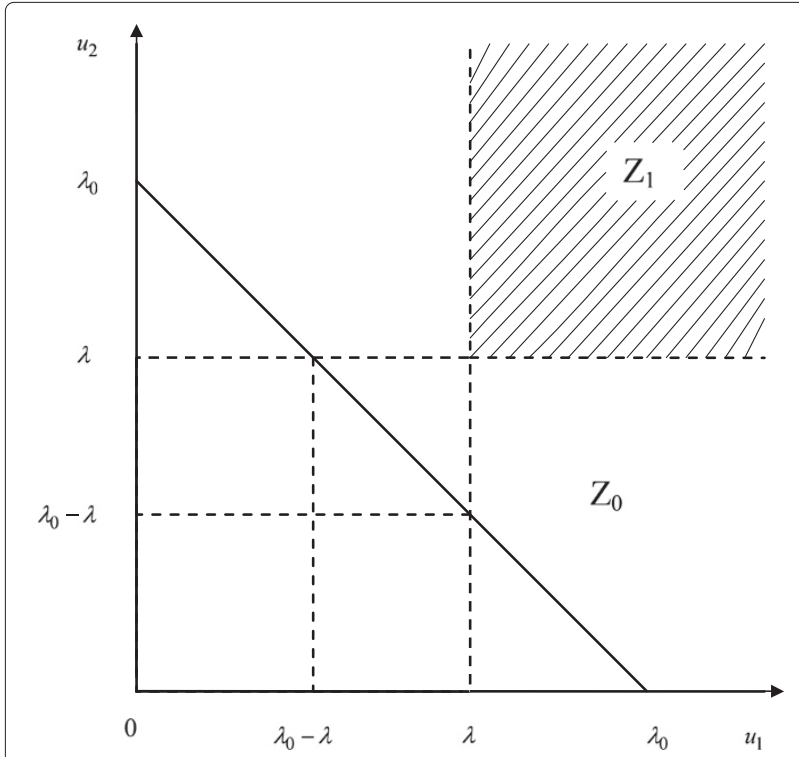

Figure 4 Decision regions for detection and non-detection of the primary signal for $\lambda_{\mathbf{0}}<\mathbf{2} \lambda$. Shaded region $Z_{1}$ corresponds to the detection region of the primary signal and unshaded region corresponds to $Z_{0}$ which denotes the region of non-detection of the primary signal.

\subsection{Optimization of the proposed selective soft combining scheme}

In this subsection, we will discuss the optimization of the local and global thresholds of the proposed soft combining scheme. The optimization is divided into three different parts, i.e., $\lambda_{0}>2 \lambda, \lambda_{0}=2 \lambda$, and $\lambda_{0}<2 \lambda$.

3.3.1 Optimization of thresholds for $\lambda_{0}>2 \lambda$

By partially differentiating Eq. (20) with respect to (w.r.t.) the normalized global threshold $\lambda_{0 n}$, we get

$$
\frac{\partial P_{e_{1}}}{\partial \lambda_{0 n}}=\frac{1}{2} \frac{\partial P_{f_{1}}}{\partial \lambda_{0 n}}+\frac{1}{2} \frac{\partial P_{m_{1}}}{\partial \lambda_{0 n}},
$$

where

$$
\begin{aligned}
\frac{\partial P_{f_{1}}}{\partial \lambda_{0 n}}= & +\frac{1}{\Gamma(2 L)} \exp \left(-L \lambda_{0 n}\right) \sum_{l=0}^{2 L-1} \sum_{i=0}^{l}\left(\begin{array}{l}
l \\
i
\end{array}\right) \\
& \times \frac{(-1)^{l-i} L^{l+2 L}\left[i \lambda_{0 n}^{i-1}\left(\lambda_{0 n}-\lambda_{n}\right)^{l+2 L-i}-i \lambda_{0 n}^{i-1} \lambda_{n}^{l+2 L-i}\right]}{l !(l+2 L-i)} \\
& -\frac{L}{\Gamma(2 L)} \exp \left(-L \lambda_{0 n}\right) \sum_{l=0}^{2 L-1} \sum_{i=0}^{l}\left(\begin{array}{l}
l \\
i
\end{array}\right) \\
& \times \frac{(-1)^{l-i} L^{l+2 L} \lambda_{0 n}^{i}\left[\left(\lambda_{0 n}-\lambda_{n}\right)^{l+2 L-i}-\lambda_{n}^{l+2 L-i}\right]}{l !(l+2 L-i)} \\
& +\frac{1}{\Gamma(2 L)} \exp \left(-L \lambda_{0 n}\right) \sum_{l=0}^{2 L-1} \sum_{i=0}^{l}\left(\begin{array}{l}
l \\
i
\end{array}\right) \\
& \times \frac{(-1)^{l-i} L^{l+2 L} \lambda_{0 n}^{i}\left(\lambda_{0 n}-\lambda_{n}\right)^{l+2 L-i-1}}{l !} \\
& -\frac{L \Gamma\left(2 L, L \lambda_{n}\right)\left(L \lambda_{0 n}-L \lambda_{n}\right)^{2 L-1} \exp \left(-\left(L \lambda_{0 n}-L \lambda_{n}\right)\right)}{[\Gamma(2 L)]^{2}}
\end{aligned}
$$

and

$$
\begin{aligned}
\frac{\partial P_{m_{1}}}{\partial \lambda_{0 n}}= & +\frac{1}{\Gamma(2 L)} \exp \left(-\frac{L \lambda_{0 n}}{1+\gamma}\right) \sum_{l=0}^{2 L-1} \sum_{i=0}^{l}\left(\begin{array}{l}
l \\
i
\end{array}\right) \\
& \times \frac{(-1)^{l-i} L^{l+2 L}\left[i \lambda_{0 n}^{i-1}\left(\lambda_{0 n}-\lambda_{n}\right)^{l+2 L-i}-i \lambda_{0 n}^{i-1} \lambda_{n}^{l+2 L-i}\right]}{l !(l+2 L-i)(1+\gamma)^{l+2 L}} \\
& -\frac{L}{\Gamma(2 L)} \exp \left(-\frac{L \lambda_{0 n}}{1+\gamma}\right) \sum_{l=0}^{2 L-1} \sum_{i=0}^{l}\left(\begin{array}{l}
l \\
i
\end{array}\right) \\
& \times \frac{(-1)^{l-i} L^{l+2 L} \lambda_{0 n}^{i}\left[\left(\lambda_{0 n}-\lambda_{n}\right)^{l+2 L-i}-\lambda_{n}^{l+2 L-i}\right]}{l !(l+2 L-i)(1+\gamma)^{l+2 L+1}} \\
& +\frac{1}{\Gamma(2 L)} \exp \left(-\frac{L \lambda_{0 n}}{1+\gamma}\right)^{2 L-1} \sum_{l=0}^{l} \sum_{i=0}^{l}\left(\begin{array}{l}
l \\
i
\end{array}\right) \\
& \times \frac{(-1)^{l-i} L^{l+2 L} \lambda_{0 n}^{i}\left(\lambda_{0 n}-\lambda_{n}\right)^{l+2 L-i-1}}{l !(1+\gamma)^{l+2 L+i}} \\
& -\frac{L \Gamma\left(2 L, \frac{L \lambda_{n}}{1+\gamma}\right)\left(\frac{L \lambda_{0 n}-L \lambda_{n}}{1+\gamma}\right)^{2 L-1} \exp \left(-\frac{L \lambda_{0 n}-L \lambda_{n}}{1+\gamma}\right)}{(1+\gamma)[\Gamma(2 L)]^{2}}
\end{aligned}
$$


We can solve Eq. (25) numerically to find the optimum value of $\lambda_{0 n}$. In order to obtain optimal value of local normalized threshold $\lambda_{n}$, partially differentiate Eq. (20) w.r.t. $\lambda_{n}$, to get

$$
\frac{\partial P_{e_{1}}}{\partial \lambda_{n}}=\frac{1}{2} \frac{\partial P_{f_{1}}}{\partial \lambda_{n}}+\frac{1}{2} \frac{\partial P_{m_{1}}}{\partial \lambda_{n}},
$$

where

$$
\begin{aligned}
\frac{\partial P_{f_{1}}}{\partial \lambda_{n}}= & -\frac{1}{\Gamma(2 L)} \exp \left(-L \lambda_{0 n}\right) \sum_{l=0}^{2 L-1} \sum_{i=0}^{l}\left(\begin{array}{l}
l \\
i
\end{array}\right) \\
& \times \frac{(-1)^{l-i} L^{l+2 L} \lambda_{0 n}^{i}\left[\left(\lambda_{0 n}+\lambda_{n}\right)^{l+2 L-i-1}-\lambda_{n}^{l+2 L-i-1}\right]}{l !} \\
& +\frac{L^{2 L} \Gamma\left(2 L, L \lambda_{n}\right)\left(\lambda_{0 n}-\lambda_{n}\right)^{2 L-1} \exp \left(-\left(L \lambda_{0 n}-L \lambda_{n}\right)\right)}{[\Gamma(2 L)]^{2}} \\
& -\frac{L^{2 L} \Gamma\left(2 L, L\left(\lambda_{0 n}-\lambda_{n}\right)\right) \lambda_{n}^{2 L-1} \exp \left(-L \lambda_{n}\right)}{[\Gamma(2 L)]^{2}}
\end{aligned}
$$

and

$$
\begin{aligned}
\frac{\partial P_{m_{1}}}{\partial \lambda_{n}}= & -\frac{1}{\Gamma(2 L)} \exp \left(-\frac{L \lambda_{0 n}}{1+\gamma}\right) \sum_{l=0}^{2 L-1} \sum_{i=0}^{l}\left(\begin{array}{l}
l \\
i
\end{array}\right) \\
& \times \frac{(-1)^{l-i} L^{l+2 L} \lambda_{0 n}^{i}\left[\left(\lambda_{0 n}+\lambda_{n}\right)^{l+2 L-i-1}-\lambda_{n}^{l+2 L-i-1}\right]}{l !(1+\gamma)^{l+2 L}} \\
& +\frac{L^{2 L} \Gamma\left(2 L, \frac{L \lambda_{n}}{1+\gamma}\right)\left(\lambda_{0 n}-\lambda_{n}\right)^{2 L-1} \exp \left(-\frac{L \lambda_{0 n}-L \lambda_{n}}{1+\gamma}\right)}{(1+\gamma)^{2 L}[\Gamma(2 L)]^{2}} \\
& -\frac{L^{2 L} \Gamma\left(2 L, \frac{L\left(\lambda_{0 n}-\lambda_{n}\right)}{1+\gamma}\right) \lambda_{n}^{2 L-1} \exp \left(-\frac{L \lambda_{n}}{1+\gamma}\right)}{(1+\gamma)^{2 L}[\Gamma(2 L)]^{2}}
\end{aligned}
$$

For finding an optimal value of $\lambda_{n}$, we need to numerically solve Eq. (28). We can also numerically find the joint values of $\lambda_{0 n}$ and $\lambda_{n}$ such that total probability of error is minimized [28].

Let us find the optimum value of the global threshold $\lambda_{0 n}$ with a single received data sample and the local threshold in each CR set to zero, i.e., $\lambda_{n}=0$. On partially differentiating Eq. (20) w.r.t. $\lambda_{0 n}$ when $\lambda_{n}=0$ and setting the result to zero, we get

$$
\lambda_{0 n}^{*}=4\left(1+\frac{1}{\gamma}\right) \ln (1+\gamma),
$$

where $\lambda_{0 n}^{*}$ denotes the optimal value of the normalized global threshold in the proposed scheme. It will be shown by simulations in Section 5 that the minimum probability of error in detection of a spectrum hole is achieved for $\lambda_{n}=0$.

\subsubsection{Optimization of thresholds for $\lambda_{0} \leq 2 \lambda$}

Since the total probability of error for $\lambda_{0}=2 \lambda$ and $\lambda_{0}<$ $2 \lambda$ is the same, we can consider them together as $\lambda_{0} \leq$ $2 \lambda$ for optimization of the thresholds. In order to obtain the optimal value of $\lambda_{n}$, we need to partially differentiate Eq. (24) w.r.t. $\lambda_{n}$, to get

$$
\begin{aligned}
\frac{\partial P_{e_{2}}}{\partial \lambda_{n}}=\frac{2 L^{2 L} \lambda_{n}^{2 L-1}}{\Gamma(2 L)} & \left(\frac{\exp \left(-\frac{L \lambda_{n}}{1+\gamma}\right) \Gamma\left(2 L, \frac{L \lambda_{n}}{1+\gamma}\right)}{(1+\gamma)^{2 L}}\right. \\
& \left.-\exp \left(-L \lambda_{n}\right) \Gamma\left(2 L, L \lambda_{n}\right)\right) .
\end{aligned}
$$

We can numerically solve Eq. (32) to find the optimized value of $\lambda_{n}$.

\subsection{Performance analysis of semi-selective combining scheme}

In the semi-selective combining scheme, the FC combines the energy estimates of the CRs corresponding to detection region $Z_{1}$ for the condition that $u_{1}>\lambda, u_{2}<\lambda$, and $u_{1}+u_{2}>\lambda_{0}, u_{1}<\lambda, u_{2}>\lambda$, and $u_{1}+u_{2}>\lambda_{0}$, and $u_{1}<\lambda, u_{2}<\lambda$, and $u_{1}+u_{2}>\lambda_{0}$ also in addition to $u_{1}>\lambda, u_{2}>\lambda$, and $u_{1}+u_{2}>\lambda_{0}$ considered in previous subsections. By following the procedure given in Section 3.1, we can split the analysis into three parts $\lambda_{0}>2 \lambda, \lambda_{0}=2 \lambda$, and $\lambda_{0}<2 \lambda$ and obtain the probability of false alarm and missed detection for these three conditions as follows ${ }^{\mathrm{a}}$.

\subsubsection{Probability of false alarm and missed detection for $\lambda_{0}>2 \lambda$}

When $u_{1}>\lambda, u_{2}<\lambda, u_{1}+u_{2}>\lambda_{0}$, and $\lambda_{0}>2 \lambda$, the probability of false alarm and missed detection will be

$$
\begin{aligned}
P_{f_{1}}^{(1)}= & \frac{1}{[\Gamma(2 L)]^{2}} \Gamma\left(2 L, \frac{L\left(\lambda_{0}-\lambda\right)}{\sigma_{n}^{2}}\right) \gamma\left(2 L, \frac{L \lambda}{\sigma_{n}^{2}}\right) \\
& -\frac{1}{[\Gamma(2 L)]^{2}} \int_{\frac{L\left(\lambda_{0}-\lambda\right)}{\sigma_{n}^{2}}}^{\frac{L \lambda_{0}}{\sigma_{n}^{2}}} \gamma\left(2 L, \frac{L \lambda_{0}}{\sigma_{n}^{2}}-t\right) t^{2 L-1} \exp (-t) d t . \\
P_{m_{1}}^{(1)}= & 1-\frac{1}{[\Gamma(2 L)]^{2}} \Gamma\left(2 L, \frac{L\left(\lambda_{0}-\lambda\right)}{\sigma_{h n}^{2}}\right) \gamma\left(2 L, \frac{L \lambda}{\sigma_{h n}^{2}}\right) \\
& +\frac{1}{[\Gamma(2 L)]^{2}} \int_{\frac{L\left(\lambda_{0}-\lambda\right)}{\sigma_{h n}^{2}}}^{\frac{L \lambda_{0}}{\sigma_{h n}^{2}}}\left(2 L, \frac{L \lambda_{0}}{\sigma_{h n}^{2}}-t\right) t^{2 L-1} \\
& \times \exp (-t) d t .
\end{aligned}
$$

For $u_{1}<\lambda, u_{2}>\lambda, u_{1}+u_{2}>\lambda$, and $\lambda_{0}>2 \lambda$, the false and missed detection probabilities will be found as

$$
\begin{aligned}
& P_{f_{1}}^{(2)}=\frac{1}{[\Gamma(2 L)]^{2}} \int_{0}^{\frac{L \lambda}{\sigma_{n}^{2}}} \Gamma\left(2 L, \frac{L \lambda_{0}}{\sigma_{n}^{2}}-t\right) t^{2 L-1} \exp (-t) d t, \quad(35) \\
& P_{m_{1}}^{(2)}=1-\frac{1}{[\Gamma(2 L)]^{2}} \int_{0}^{\frac{L \lambda}{\sigma_{h n}^{2}}} \Gamma\left(2 L, \frac{L \lambda_{0}}{\sigma_{h n}^{2}}-t\right) t^{2 L-1} \exp (-t) d t .
\end{aligned}
$$


In case of $u_{1}<\lambda, u_{2}<\lambda, u_{1}+u_{2}>\lambda_{0}$, and $\lambda_{0}>2 \lambda$, the detection region will be zero and probability of miss will be one.

\subsubsection{Probability of false alarm and missed detection for $\lambda_{0}=2 \lambda$}

The probability of false alarm and missed detection for $u_{1}>\lambda, u_{2}<\lambda, u_{1}+u_{2}>\lambda_{0}$, and $\lambda_{0}=2 \lambda$ will be

$$
\begin{aligned}
P_{f_{2}}^{(1)}= & \frac{1}{[\Gamma(2 L)]^{2}} \Gamma\left(2 L, \frac{L \lambda}{\sigma_{n}^{2}}\right) \gamma\left(2 L, \frac{L \lambda}{\sigma_{n}^{2}}\right) \\
& -\frac{1}{[\Gamma(2 L)]^{2}} \int_{\frac{L \lambda}{\sigma_{n}^{2}}}^{\frac{L \lambda_{0}}{\sigma_{n}^{2}}} \gamma\left(2 L, \frac{L \lambda_{0}}{\sigma_{n}^{2}}-t\right) t^{2 L-1} \exp (-t) d t \\
P_{m_{2}}^{(1)}= & 1-\frac{1}{[\Gamma(2 L)]^{2}} \Gamma\left(2 L, \frac{L \lambda}{\sigma_{h n}^{2}}\right) \gamma\left(2 L, \frac{L \lambda}{\sigma_{h n}^{2}}\right) \\
& +\frac{1}{[\Gamma(2 L)]^{2}} \int_{\frac{L \lambda}{\sigma_{h n}^{2}}}^{\frac{L \lambda_{0}}{\sigma_{h n}^{2}}} \gamma\left(2 L, \frac{L \lambda_{0}}{\sigma_{h n}^{2}}-t\right) t^{2 L-1} \exp (-t) d t .
\end{aligned}
$$

For $u_{1}<\lambda, u_{2}>\lambda, u_{1}+u_{2}>\lambda_{0}$, and $\lambda_{0}>2 \lambda$, the false and missed detection probabilities will be as follows:

$$
\begin{aligned}
& P_{f_{2}}^{(2)}=\frac{1}{[\Gamma(2 L)]^{2}} \int_{0}^{\frac{L \lambda}{\sigma_{n}^{2}}} \Gamma\left(2 L, \frac{L \lambda_{0}}{\sigma_{n}^{2}}-t\right) t^{2 L-1} \exp (-t) d t, \text { (39) } \\
& P_{m_{2}}^{(2)}=1-\frac{1}{[\Gamma(2 L)]^{2}} \int_{0}^{\frac{L \lambda}{\sigma_{h n}^{2}}} \Gamma\left(2 L, \frac{L \lambda_{0}}{\sigma_{h n}^{2}}-t\right) t^{2 L-1} \exp (-t) d t .
\end{aligned}
$$

When $u_{1}<\lambda, u_{2}<\lambda, u_{1}+u_{2}>\lambda_{0}$, and $\lambda_{0}>2 \lambda$, the detection region will be zero and probability of miss will be one.

\subsubsection{Probability of false alarm and missed detection for \\ $\lambda_{0}<2 \lambda$}

The probability of false alarm and missed detection for $u_{1}>\lambda, u_{2}<\lambda, u_{1}+u_{2}>\lambda_{0}$, and $\lambda_{0}=2 \lambda$ are

$$
\begin{aligned}
P_{f_{3}}^{(1)}= & \frac{1}{[\Gamma(2 L)]^{2}} \Gamma\left(2 L, \frac{L \lambda}{\sigma_{n}^{2}}\right) \gamma\left(2 L, \frac{L \lambda}{\sigma_{n}^{2}}\right) \\
- & \frac{1}{[\Gamma(2 L)]^{2}} \int_{\frac{L \lambda}{\sigma_{n}^{2}}}^{\frac{L \lambda_{0}}{\sigma_{n}^{2}}} \Gamma\left(2 L, \frac{L \lambda_{0}}{\sigma_{n}^{2}}-t\right) t^{2 L-1} \exp (-t) d t \\
P_{m_{3}}^{(1)}= & 1-\frac{1}{[\Gamma(2 L)]^{2}} \Gamma\left(2 L, \frac{L \lambda}{\sigma_{h n}^{2}}\right) \gamma\left(2 L, \frac{L \lambda}{\sigma_{h n}^{2}}\right) \\
& +\frac{1}{[\Gamma(2 L)]^{2}} \int_{\frac{L \lambda}{\sigma_{h n}^{2}}}^{\frac{L \lambda_{0}}{\sigma_{h n}^{2}}}\left(2 L, \frac{L \lambda_{0}}{\sigma_{h n}^{2}}-t\right) t^{2 L-1} \exp (-t) d t
\end{aligned}
$$

The false and missed detection probabilities for $u_{1}<\lambda$, $u_{2}>\lambda, u_{1}+u_{2}>\lambda_{0}$, and $\lambda_{0}>2 \lambda$ can be obtained as

$$
P_{f_{3}}^{(2)}=\frac{1}{[\Gamma(2 L)]^{2}} \int_{0}^{\frac{L\left(\lambda_{0}-\lambda\right)}{\sigma_{n}^{2}}} \Gamma\left(2 L, \frac{L \lambda_{0}}{\sigma_{n}^{2}}-t\right) t^{2 L-1} \exp (-t) d t,
$$

$$
P_{m_{3}}^{(2)}=1-\frac{1}{[\Gamma(2 L)]^{2}} \int_{0}^{\frac{L\left(\lambda_{0}-\lambda\right)}{\sigma_{h n}^{2}}} \Gamma\left(2 L, \frac{L \lambda_{0}}{\sigma_{h n}^{2}}-t\right) t^{2 L-1} \exp (-t) d t .
$$

In the case of $u_{1}<\lambda, u_{2}<\lambda, u_{1}+u_{2}>\lambda$, and $\lambda_{0}>2 \lambda$, the false and missed detection probabilities will be

$$
\begin{aligned}
P_{f_{3}}^{(3)}= & -\frac{1}{[\Gamma(2 L)]^{2}} \Gamma\left(2 L, \frac{L\left(\lambda_{0}-\lambda\right)}{\sigma_{n}^{2}}\right) \Gamma\left(2 L, \frac{L \lambda}{\sigma_{n}^{2}}\right) \\
& +\frac{1}{[\Gamma(2 L)]^{2}}\left[\Gamma\left(2 L, \frac{L \lambda}{\sigma_{n}^{2}}\right)\right]^{2} \\
& +\frac{1}{[\Gamma(2 L)]^{2}} \int_{\frac{L\left(\lambda_{0}-\lambda\right)}{\sigma_{n}^{2}}}^{\frac{L \lambda}{\sigma^{2}}} \gamma\left(2 L, \frac{L \lambda_{0}}{\sigma_{n}^{2}}-t\right) t^{2 L-1} \exp (-t) d t, \\
P_{m_{3}}^{(3)}= & 1+\frac{1}{[\Gamma(2 L)]^{2}} \Gamma\left(2 L, \frac{L\left(\lambda_{0}-\lambda\right)}{\sigma_{h n}^{2}}\right) \Gamma\left(2 L, \frac{L \lambda}{\sigma_{h n}^{2}}\right) \\
& -\frac{1}{[\Gamma(2 L)]^{2}}\left[\Gamma\left(2 L, \frac{L \lambda}{\sigma_{h n}^{2}}\right)\right]^{2} \\
& -\frac{1}{[\Gamma(2 L)]^{2}} \int_{\frac{L\left(\lambda_{0}-\lambda\right)}{\sigma_{h n}^{2}}}^{\frac{L \lambda}{\sigma_{h n}^{2}}} \gamma\left(2 L, \frac{L \lambda_{0}}{\sigma_{h n}^{2}}-t\right) t^{2 L-1} \exp (-t) d t .
\end{aligned}
$$

The semi-selective soft combining scheme can be optimized by the procedure given in Section 3.2, and optimized values of the local and global thresholds can be obtained by the total probability of error minimization criterion.

\section{Soft and hard combination schemes for more than two CRs}

It can be seen from the discussion above that analysis of the proposed soft combining scheme is very complex in the case of more than two users. Hence, it is very difficult to find the optimum local and global thresholds for a general collaborative soft combining scheme with more than two CRs. Therefore, in the case of more than two CRs, we can assume that the FC does not have information about the local threshold $\lambda$. Therefore, the FC cannot use selective combining discussed in Section 3.2. 


\subsection{Non-selective soft combination scheme for more than two CRs}

In the non-selective soft combining scheme for more than two users, the FC always combines the energy estimates of all CRs for taking a decision. When the FC does not use the local threshold for decision making, the nonselective soft combining scheme is similar to the scheme discussed in $[4,13]$ for arbitrary number of CRs. However, in $[4,13]$, the NP criterion is used for finding the optimized value of the global threshold. Moreover, the existing schemes $[4,13]$ consider a slowly varying Rayleigh fading channel for analysis. It can be seen from Eq. (1) that we consider a fast fading Rayleigh channel between the PU and CRs in our analysis. In addition, it can be seen from Section 3.3 that we use the total probability of error for finding the optimized value of the threshold. Therefore, we need to derive the expression of the total probability of error for the conventional non-selective soft combining scheme of $[4,13]$, and based on that, we can find a closed form expression of the optimal value of the global threshold.

In the non-selective soft combining scheme, each CR forwards the energy estimate over noiseless channel to the $\mathrm{FC}$, and the FC takes the decision about the spectrum hole based on the global threshold $\lambda_{0}$.

Let the number of secondary users or CRs be denoted by $K$. Each secondary user calculates a summary statistics $u_{i}$ over a finite detection interval of $L$ samples as

$$
u_{i}=\frac{1}{L} \sum_{k=0}^{L-1}\left|y_{i}(k)\right|^{2}
$$

where $i=1,2, \ldots, K$. The summary statistics are transmitted to the FC through a lossless control channel using orthogonal time slots or orthogonal frequency bands. The FC computes a global test statistics as

$$
U=\sum_{i=1}^{K} u_{i}
$$

which is compared with a predefined threshold $\lambda_{0}$ to make a decision about the presence of the PU. It can be noted from Eq. (48) that the process of combining summary statistics is similar to equal-gain combining.

From Eq. (48), the c.f. of $U$ in the case of hypotheses $H_{0}$ and $H_{1}$ will be

$$
\psi_{U \mid H_{0}}(j \omega)=\frac{1}{\left(1-\frac{j \omega \sigma_{n}^{2}}{L}\right)^{K L}}
$$

and

$$
\psi_{U \mid H_{1}}(j \omega)=\frac{1}{\left(1-\frac{j \omega \sigma_{h n}^{2}}{L}\right)^{K L}},
$$

respectively. By taking the inverse Fourier transform of Eqs. (49) and (50), the p.d.f.s of $U$ under hypotheses $H_{0}$ and $H_{1}$ can be obtained as

$$
f_{U \mid H_{0}}(x)=\frac{L^{K L} \exp \left(-\frac{x L}{\sigma_{n}^{2}}\right) x^{K L-1}}{\sigma_{n}^{2 K L} \Gamma(K L)}
$$

and

$$
f_{U \mid H_{1}}(x)=\frac{L^{K L} \exp \left(-\frac{x L}{\sigma_{h n}^{2}}\right) x^{K L-1}}{\sigma_{h n}^{2 K L} \Gamma(K L)},
$$

respectively. The decision rule in the $\mathrm{FC}$ is

$$
U=\sum_{i=1}^{K} u_{i} \underset{\mathrm{PU} \text { absent }}{\stackrel{\mathrm{PU} \text { present }}{\gtrless}} \lambda_{0} .
$$

From Eqs. (51) and (52), we can obtain the probability of false alarm $P_{f}$ in $\mathrm{FC}$ as

$$
P_{f}=\int_{\lambda_{0}}^{\infty} f_{U \mid H_{0}}(x) d x=\frac{\Gamma\left(K L, L \lambda_{0 n}\right)}{\Gamma(K L)}
$$

and the probability of missed detection $P_{m}$ in the FC as

$$
P_{m}=\int_{0}^{\lambda_{0}} f_{U \mid H_{1}}(x) d x=1-\frac{\Gamma\left(K L, \frac{L \lambda_{0 n}}{1+\gamma}\right)}{\Gamma(K L)} .
$$

The total error probability for equiprobable hypotheses is expressed from Eqs. (54) and (55) by

$$
P_{e}=\frac{P_{f}+P_{m}}{2}=\frac{1}{2}-\frac{\Gamma\left(K L, \frac{L \lambda_{0 n}}{1+\gamma}\right)}{2 \Gamma(K L)}+\frac{\Gamma\left(K L, L \lambda_{0 n}\right)}{2 \Gamma(K L)} .
$$

Next, the optimal value of $\lambda_{0 n}$ can be found by partially differentiating Eq. (56) w.r.t. $\lambda_{0 n}$ and setting the result to zero. The partial derivative of Eq. (56) w.r.t. $\lambda_{0 n}$ will be

$$
\frac{\partial P_{e}}{\partial \lambda_{0 n}}=\frac{L^{K L} \lambda_{0 n}^{K L-1} \exp \left(-\frac{L \lambda_{0 n}}{1+\gamma}\right)}{2(1+\gamma)^{K L} \Gamma(K L)}-\frac{L^{K L} \lambda_{0 n}^{K L-1} \exp \left(-L \lambda_{0 n}\right)}{2 \Gamma(K L)}
$$

By equating Eq. (57) to zero and after some manipulations, we get the closed form expression of the optimized normalized global threshold as

$$
\lambda_{0 n}^{*}=K\left(1+\frac{1}{\gamma}\right) \ln (1+\gamma) .
$$

\subsection{One-bit hard combination scheme}

In [7], a non-selective 1-bit hard combination scheme for arbitrary number of CRs is studied. Since the detection 
error analysis of a non-selective 1-bit hard combination scheme over slowly fading Rayleigh channels is performed in [7], we will find the total probability of error and optimized value of the local threshold for fast fading Rayleigh channels for comparison with the proposed selective combining schemes. In hard combining scheme, each CR takes a binary decision about the presence or absence of the primary signal using energy detector. The FC combines these binary decisions to find the presence or absence of the PU as follows [7]:

$$
D=\sum_{i=1}^{K} d_{i}
$$

where $\mathrm{D}$ is the sum of the all 1-bit decisions from the CRs. Let $n, n \leq K$ corresponds to a number of cooperating CRs out of $K$ CRs. The FC uses a majority rule for deciding the presence or absence of the PU. As per the majority decision rule if $D$ is greater than $n$, then hypothesis $\mathcal{H}_{1}$ holds otherwise the hypothesis $\mathcal{H}_{0}$ is true. The hypotheses $\mathcal{H}_{0}$ and $\mathcal{H}_{1}$ can be written as [7]

$$
\begin{aligned}
& \mathcal{H}_{0}: \quad D<n, \quad \text { if PU is absent, } \\
& \mathcal{H}_{1}: \quad D \geq n, \quad \text { if PU is present. }
\end{aligned}
$$

The p.d.f.s of $u_{i}$ under hypotheses $\mathcal{H}_{0}$ and $\mathcal{H}_{1}$ can be obtained after some algebra as

$$
f_{u_{i} \mid \mathcal{H}_{0}}(x)=\frac{L^{L} \exp \left(-\frac{x L}{\sigma_{n}^{2}}\right) x^{L-1}}{\sigma_{n}^{2 L} \Gamma(L)}
$$

and

$$
f_{u_{i} \mid \mathcal{H}_{1}}(x)=\frac{L^{L} \exp \left(-\frac{x L}{\sigma_{h n}^{2}}\right) x^{L-1}}{\sigma_{h n}^{2 L} \Gamma(L)},
$$

respectively. The decision rule in the $i$-th, $i=1, \ldots, K, \mathrm{CR}$ is

$$
u_{i} \underset{\text { PU absent }}{\gtrless} \lambda \text {. }
$$

From Eqs. (62), (63), and (64), we get the probability of false alarm $P_{f}$ and probability of missed detection $P_{m}$ in each CR as

$$
\begin{aligned}
& P_{f}=\frac{\Gamma\left(L, L \lambda_{n}\right)}{\Gamma(L)}, \\
& P_{m}=1-\frac{\Gamma\left(L, \frac{L \lambda_{n}}{1+\gamma}\right)}{\Gamma(L)} .
\end{aligned}
$$

The probability of false alarm $P_{F}$ in the FC for cooperative sensing will be [7]

$$
P_{F}=\operatorname{Pr}\left(\mathcal{H}_{1} \mid \mathcal{H}_{0}\right)=\sum_{l=n}^{K}\left(\begin{array}{c}
K \\
l
\end{array}\right) P_{f}^{l}\left(1-P_{f}\right)^{K-l}
$$

and the probability of missed detection $P_{M}$ in $\mathrm{FC}$ will be [7]

$$
P_{M}=\operatorname{Pr}\left(\mathcal{H}_{0} \mid \mathcal{H}_{1}\right)=1-\sum_{l=n}^{K}\left(\begin{array}{c}
K \\
l
\end{array}\right)\left(1-P_{m}\right)^{l}\left(P_{m}\right)^{K-l}
$$

The total error rate of the hard combining-based cooperative scheme will be the sum of $P_{F}$ and $P_{M}$. The optimal number of CRs and optimized value of the local threshold can be calculated by minimizing the total error rate as shown in [7].

\section{Numerical results}

We consider a cognitive system with one PU, two CRs, and a FC for simulations. Moreover, we use local and global thresholds normalized with noise variance $\sigma_{n}^{2}$ in simulations. In Figure 5, the total probability of error versus normalized global threshold $\lambda_{0 n}$ plots of the proposed selective soft combining scheme with different number of received samples $L=1,2,3,4$ and PU-CR link SNR=0, 5 $\mathrm{dB}$ are shown. It can be seen from Figure 5 that the total probability of error is a convex function of the normalized global threshold in the proposed selective soft combining scheme. The total probability of error is calculated from Eq. (20). It can be observed from Figure 5 that by increasing the number of samples and SNR, very low value of the total probability of error can be achieved.

Figure 6 shows that the total probability of error obtained from Eq. (24) is a convex function of normalized local threshold in the proposed selective soft combining scheme when $\lambda_{0}<2 \lambda$. Moreover, it can be seen from Figure 6 that the total probability of error reduces by increasing number of data samples and SNR of the PU-CR link. It can be observed from Figures 7, 8, and 9 that for a single received data sample, it is possible to minimize the total probability of error at a given SNR of the PU-CR link by an optimized global threshold in the proposed selective soft combining scheme.

Optimized values of the global thresholds for the proposed selective soft combining scheme and non-selective combining scheme (Sections 3.3 and 4.1) are calculated for each SNR values from Eqs. (31) and (58), respectively. Whereas, the global thresholds for the hard combining scheme (Section 4.2) are calculated numerically at each SNR value using Eqs. (67) and (68). Table 1 shows the 


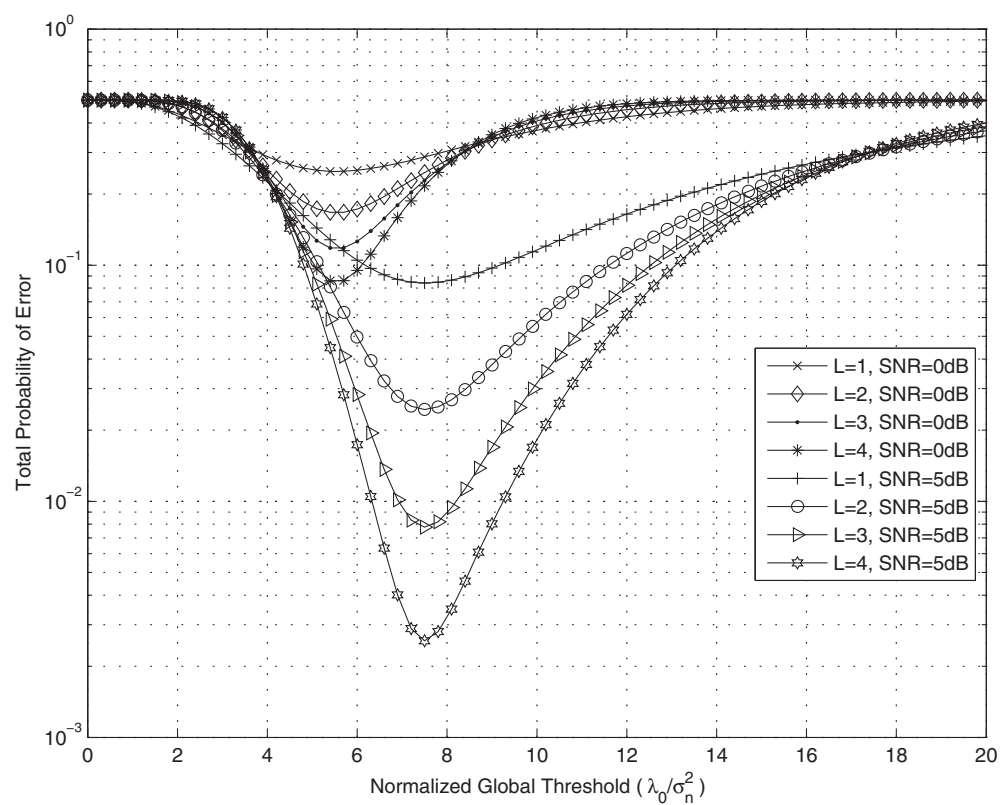

Figure 5 Total probability of error versus normalized global threshold plots of the proposed selective soft combining scheme. With two $\mathrm{CRs} ; \lambda_{n}=0$; different number of samples $L=1,2,3$, 4; and $\mathrm{SNR}=0,5 \mathrm{~dB}$.

minimum value of total probability of error at optimal value of the normalized global threshold of the proposed selective soft combining scheme with two CRs, $L=1$, and $\mathrm{SNR}=-10,0,10 \mathrm{~dB}$ for different numbers of normalized local threshold. It can be concluded from Table 1 that as the $\lambda_{n}$ is increased to 4.0 , a given SNR total probability of error also increases.

In Figure 10, we have plotted the total probability of error versus SNR of the PU-CR link plots for the proposed selective soft combining scheme, non-selective

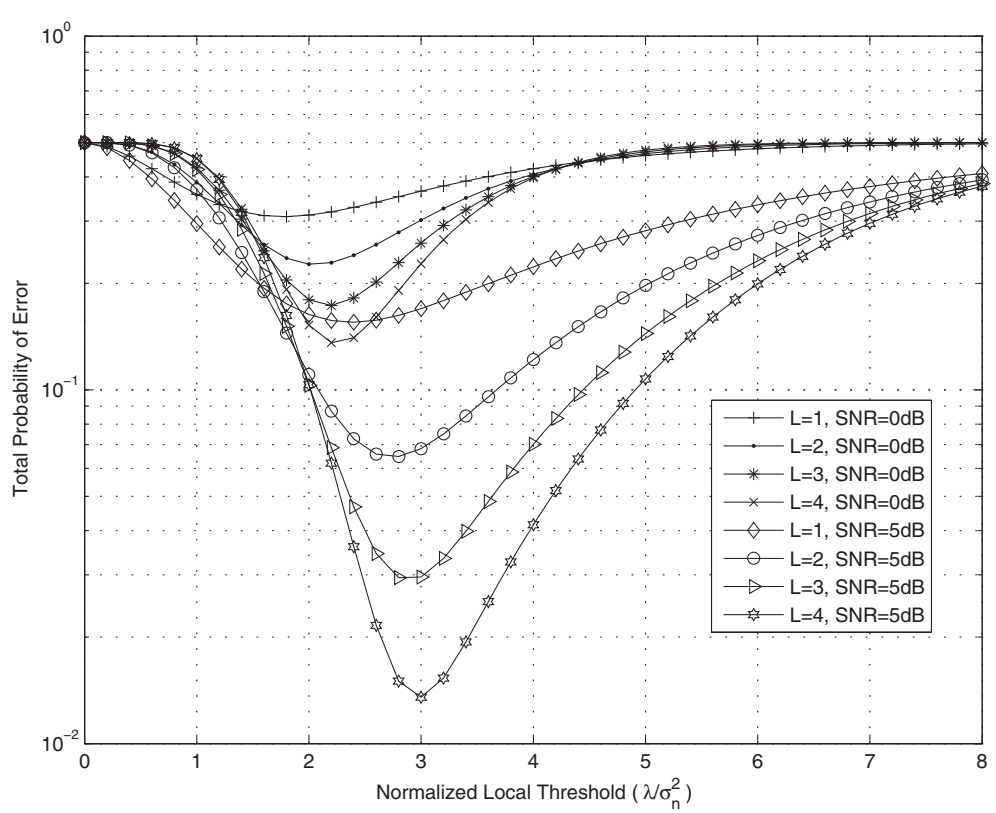

Figure 6 Total probability of error versus normalized local threshold plots of the proposed selective soft combining scheme. With two CRs; $\lambda_{0}<2 \lambda ; L=1,2,3,4$; and $S N R=0,5 \mathrm{~dB}$. 


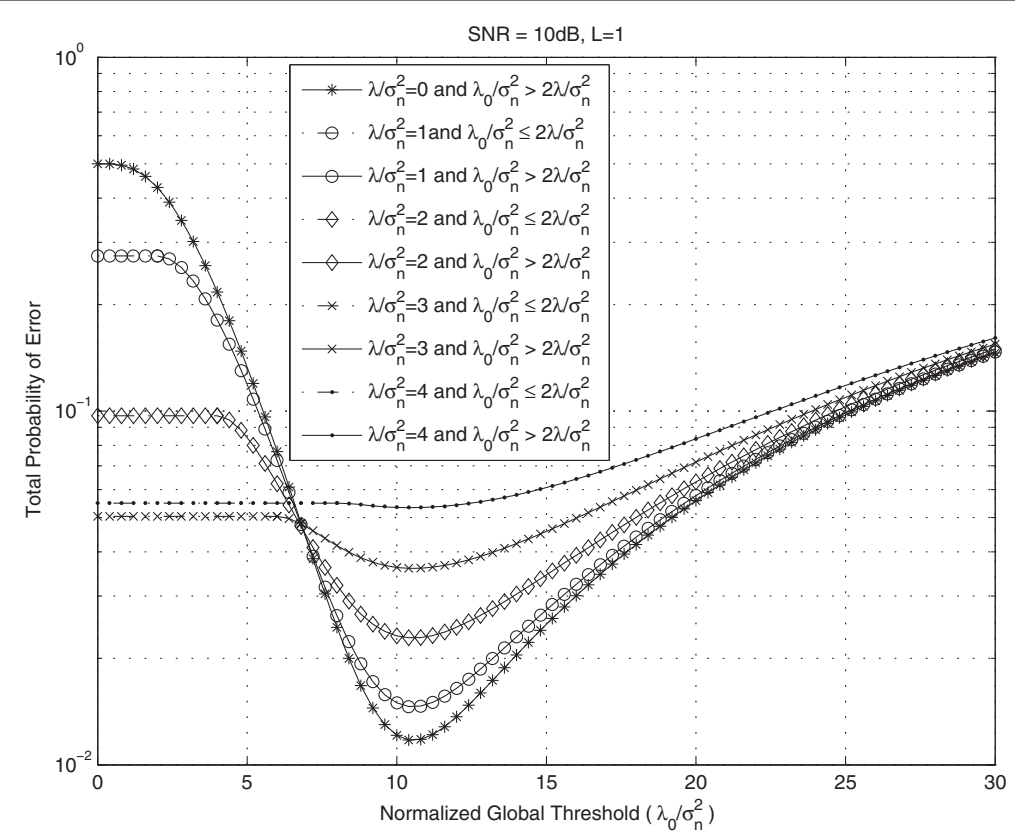

Figure 7 Total probability of error versus normalized global threshold plots of the proposed selective soft combining scheme. With two $C R s, L=1$, and $S N R=10 \mathrm{~dB}$.

soft combining scheme, and 1-bit hard combining scheme for two CRs, one received data sample, and $\lambda_{n}=0$. It can observed from Figure 10 that the proposed selective soft combining scheme significantly outperforms the non-selective soft and hard combining schemes. In order to compare the proposed selective and nonselective soft combining schemes under NP and total error minimization criterion, plots of the probability of detection of spectrum hole and probability of false alarm versus SNR of PU-CR link are shown in

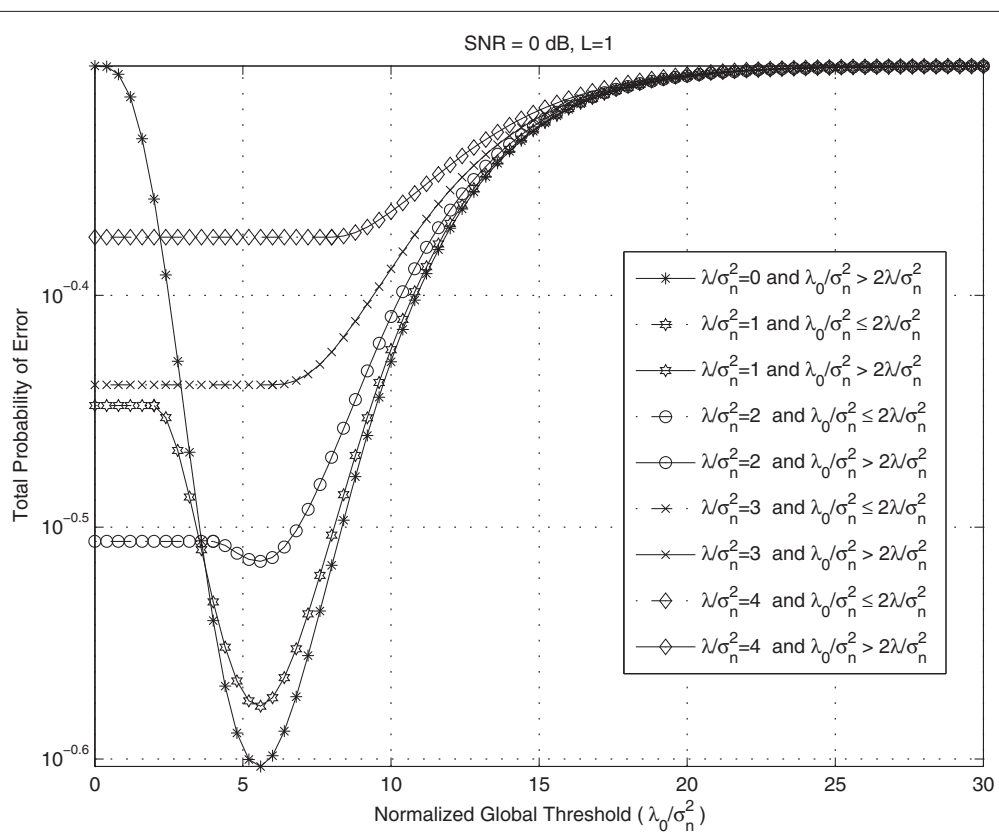

Figure 8 Total probability of error versus normalized global threshold plots of the proposed selective soft combining scheme. With two $C R s, L=1$, and $S N R=0 \mathrm{~dB}$. 


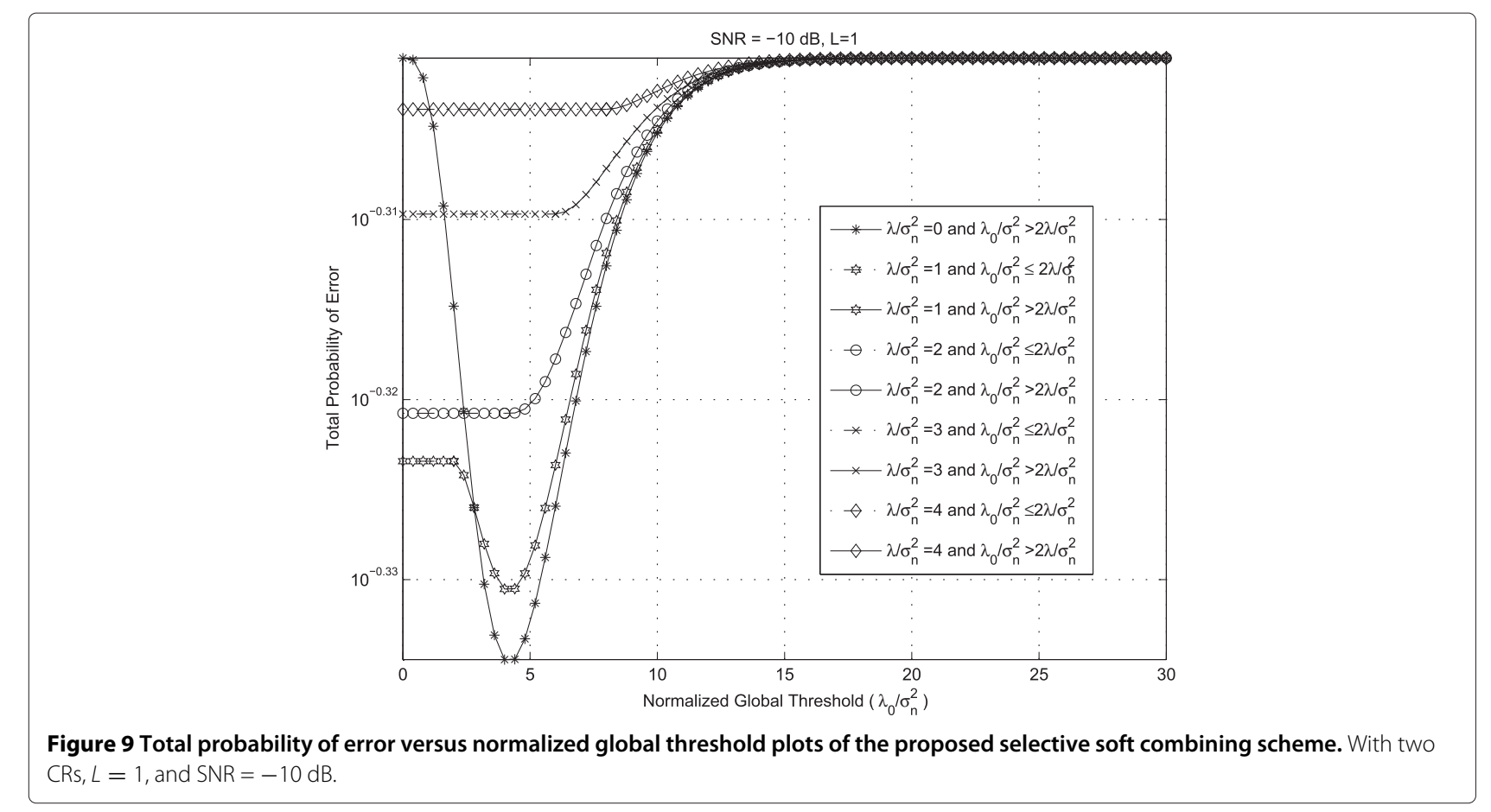

Figures 2 and 11, respectively, for a single received data sample.

Under the NP criterion, the non-selective soft combining scheme explained in Section 4.1 is optimized as discussed in $[4,13]$. It can be seen from Figure 11 that the probability of detection under the total probability of error minimization criterion is better than that obtained using the NP criterion for the selective and non-selective soft combining schemes for the considered SNR values. Moreover, under the total probability of error minimization criterion, the proposed selective soft combining scheme significantly outperforms the non-selective soft combining scheme over the range of SNR values used in Figure 11. For example, an SNR gain of approximately

Table 1 Optimal values of normalized global threshold for varying values of normalized local threshold

\begin{tabular}{ccccc}
\hline Sample no. & SNR (dB) & Total prob. of error & Normalized local threshold & \multicolumn{2}{c}{ Normalized global threshold } \\
& & & $\lambda_{n}$ & $\lambda_{0 n}^{*}$ \\
\hline 1 & -10 & 0.4630 & 0.0 & 4.4 \\
2 & -10 & 0.4672 & 1.0 & 4.4 \\
3 & -10 & 0.4778 & 2.0 & 4.4 \\
4 & -10 & 0.4901 & 3.0 & 4.4 \\
5 & -10 & 0.4967 & 4.0 & 4.4 \\
6 & 0 & 0.2493 & 0.0 & 5.6 \\
7 & 0 & 0.2647 & 1.0 & 5.6 \\
8 & 0 & 0.3057 & 2.0 & 5.6 \\
9 & 0 & 0.3642 & 3.0 & 5.6 \\
10 & 0 & 0.4218 & 4.0 & 5.6 \\
11 & 10 & 0.0118 & 0.0 & 10.4 \\
12 & 10 & 0.1462 & 1.0 & 10.4 \\
14 & 10 & 0.2286 & 2.0 & 10.4 \\
15 & 10 & 0.0359 & 3.0 & 10.4 \\
\hline
\end{tabular}




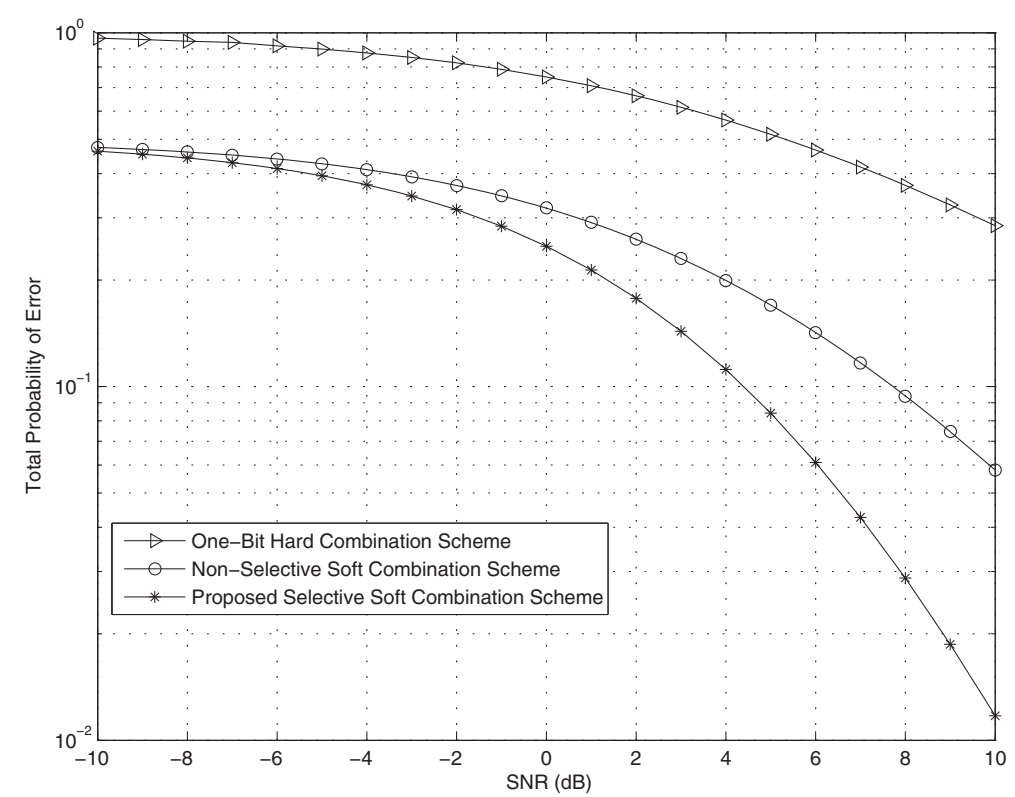

Figure 10 Total probability of error versus SNR plots. Of the 1-bit hard combination scheme, non-selective soft combination scheme, and proposed selective soft combination scheme for two cognitive radios.

$3 \mathrm{~dB}$ is achieved at the probability of detection $=0.7$ by the proposed selective scheme as compared to the non-selective scheme under the total probability of error criterion. Figure 11 shows that the proposed selective soft combining scheme under the total error probability minimization criterion provides better probability detection than the non-selective soft combining scheme using the proposed total error probability minimization and
NP criteria for the considered SNR values. The probability of false alarm versus SNR plots of the proposed selective and non-selective soft combining schemes are shown in Figure 12. It can be seen from Figure 12 that the probability of false alarm of the selective and nonselective schemes under the NP criterion is much less than 0.1 , which corresponds to the tolerable value of interference at the PU as specified by IEEE 802.22 cognitive

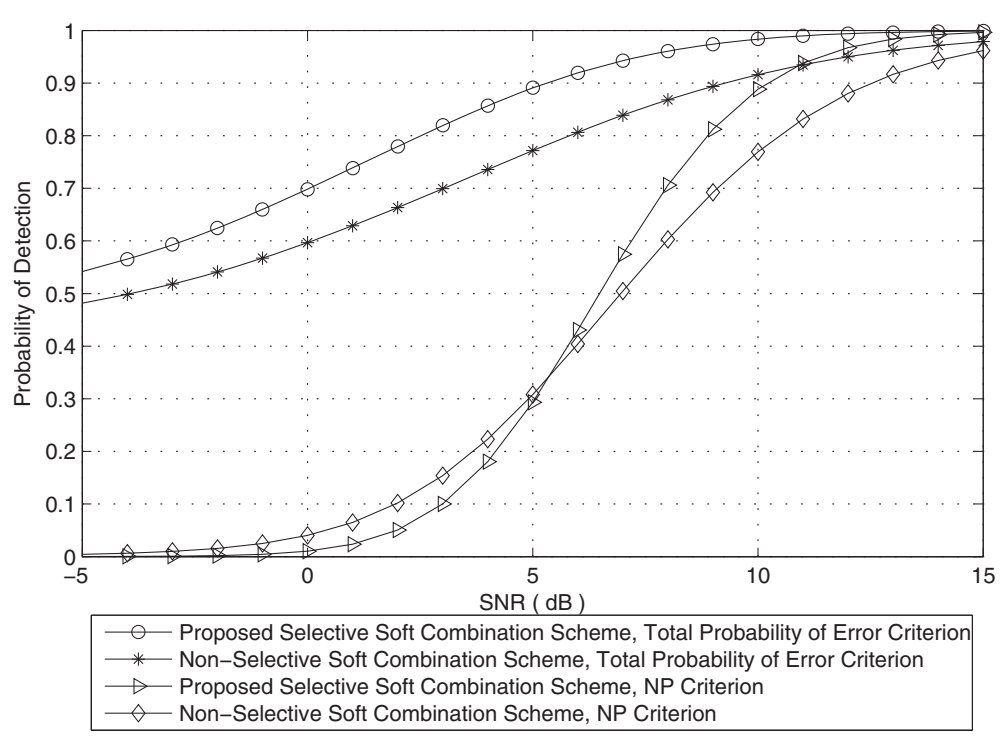

Figure 11 Probability of detection versus SNR for two cognitive radios. Of the proposed selective and non-selective soft combination scheme under NP and total probability of error minimization criterion with $L=1$. 


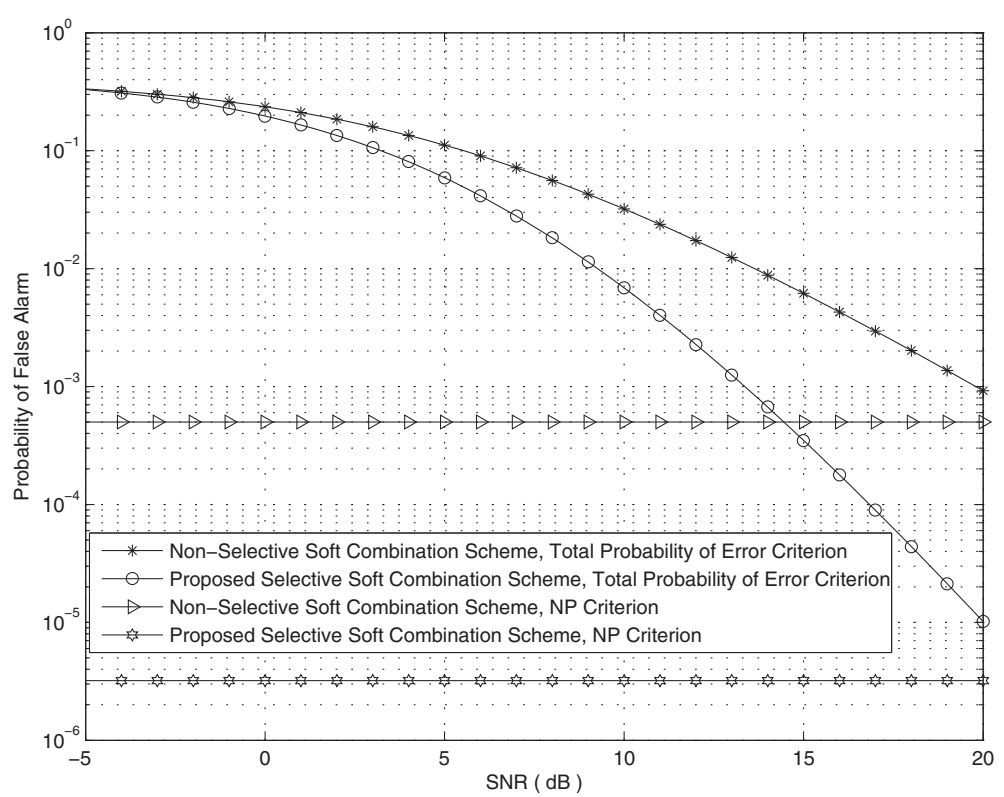

Figure 12 Probability of false alarm versus SNR plots. Of the proposed selective and non-selective soft combination scheme under the NP and total probability of error minimization criteria with $L=1$.

wireless regional area network (WRAN) standard [29]. Under the NP criterion, this very low (much lower than the tolerance level) value of the probability of false alarm is achieved at the cost of very poor probability of detection, as shown in Figure 11, which leads to significant underutilization of the available spectrum hole. Whereas, it can be seen from Figures 2 and 11 that the proposed selective combining scheme under the total error minimization criterion provides better probability of detection than the non-selective scheme using the NP criterion while keeping the probability of false alarm lower than the specified value, i.e., 0.1 for $3 \leq \mathrm{SNR} \leq 20$. Therefore, the proposed selective soft combining scheme along with the total probability of error minimization criterion is able to properly utilize a spectrum hole with interference level less than the specified value. It can be seen from Table 2 that with increasing value of $L$ of the proposed selective combining scheme, the probabilities of false alarm and missed detection decreases at given SNR $=-20,-2 \mathrm{~dB}$. Table 2 shows that requirements proposed in IEEE 802.22 WRAN is not achieved for the proposed selective combining scheme when $L$ is varied from 1 to 8 at SNR = $-20 \mathrm{~dB}$. However, at $\mathrm{SNR}=-2 \mathrm{~dB}$, the requirements can be achieved when $L=8$ with $t_{\mathrm{CDT}}=0.000128 \mathrm{~s}$, where $t_{\mathrm{CDT}}$ denotes the channel detection time, while no other processing delays are assumed. It can be noted that with increasing number of samples, the time to detect the primary signal will also increase for a given sampling frequency. In order to detect a spectrum hole quickly while satisfying IEEE 802.22 WRAN standards, we have considered finite small number of samples in our numerical results.

\section{Table 2 Effect of $L$ on the performance of cooperative spectrum sensing}

\begin{tabular}{ccccc}
\hline $\boldsymbol{L}$ & $\boldsymbol{P}_{\boldsymbol{f}}$ at SNR=-20 dB & $\boldsymbol{P}_{\boldsymbol{f}}$ at SNR=-2 dB & $\boldsymbol{P}_{\boldsymbol{m}}$ at SNR= -20 dB & $\boldsymbol{P}_{\boldsymbol{m}}$ at SNR=-2 dB \\
\hline 1 & 0.4532 & 0.2513 & 0.5390 & 0.3812 \\
2 & 0.4812 & 0.2027 & 0.5077 & 0.2917 \\
3 & 0.4963 & 0.1657 & 0.4901 & 0.2354 \\
4 & 0.5069 & 0.1369 & 0.4775 & 0.1946 \\
5 & 0.5151 & 0.1140 & 0.4673 & 0.1631 \\
6 & 0.5220 & 0.0956 & 0.4588 & 0.1380 \\
7 & 0.5280 & 0.0805 & 0.4513 & 0.1176 \\
8 & 0.5333 & 0.0681 & 0.4447 & 0.1007 \\
\hline
\end{tabular}




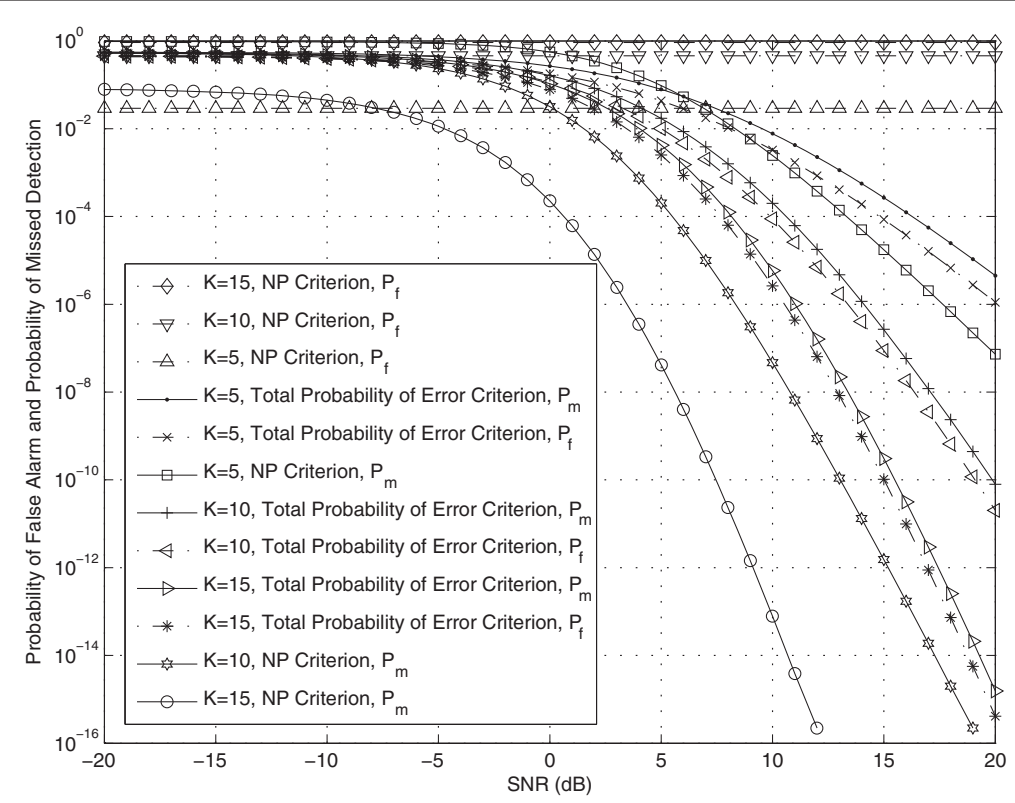

Figure 13 Probability of false alarm $\left(\boldsymbol{P}_{\boldsymbol{f}}\right)$ and probability of miss $\left(\boldsymbol{P}_{\boldsymbol{m}}\right)$ versus SNR plots. Of the non-selective soft combination scheme under the NP and total probability of error minimization criteria with $L=1$.

It can be seen from Figure 13 and Table 3 that as the number of CRs is increased from $K=5$ to $K=15$, the probability of false alarm also increases under NP criterion at $\mathrm{SNR}=10 \mathrm{~dB}$. However, probability of missed detection reduces with increasing number of CRs from $K=5$ to $K=15$ under NP criterion. It can also observed from Figure 13 and Table 3 that at $S N R=10 \mathrm{~dB}$, the probability of missed detection decreases with increasing number of CRs under total error probability criterion and NP criterion. Table 3 also shows that the probability of false alarm under total error probability criterion is much less than that under the NP criterion; hence, it indicates that the total error probability criterion performs better than NP criterion. On the other hand, this improvement in the probability of false alarm is achieved at the cost of much higher probability of missed detection. It can also be observed from Figure 13 that at a very low SNR (say $-20 \mathrm{~dB}$ ), there is almost negligible difference in the probabilities of false alarm and missed detection under NP and the total error probability criterion. As the number of CRs are increased to 15 for single sample of received signal, the values of probability of false alarm and missed detection is almost same. Figure 13 shows that at SNR $=0 \mathrm{~dB}$, the probability of missed detection $\leq 0.1$ and probability of false alarm $\leq 0.1$ under the total error probability criterion, and hence, the requirement proposed in IEEE 802.22 WRAN can be satisfied in this low SNR which is very difficult to achieve in the case of NP criterion.

\section{Conclusions}

In this paper, we have demonstrated that it is possible for a collaborative cognitive radio network to detect the spectrum hole in an optimal manner by minimizing the total probability of error in decision making with a finite number of the received data samples. It is shown by simulations that the proposed selective scheme for soft combination significantly outperforms the non-selective soft and hard combining schemes. Moreover, the total probability of error minimization criterion performs better than

Table 3 Non-selective soft combination scheme with total error probability criterion and NP criterion at SNR=10 dB and $L=1$

\begin{tabular}{ccccr}
\hline Number of CRs $(\boldsymbol{K})$ & $\begin{array}{c}\boldsymbol{P}_{\boldsymbol{f}} \\
\text { (NP criteria) }\end{array}$ & $\begin{array}{c}\boldsymbol{P}_{\boldsymbol{f}} \\
\text { (Total probability of error) }\end{array}$ & $\begin{array}{c}\boldsymbol{P}_{\boldsymbol{m}} \\
\text { (NP criteria) }\end{array}$ & $\begin{array}{c}\boldsymbol{P}_{\boldsymbol{m}} \\
\text { (Total probability of error) }\end{array}$ \\
\hline 5 & 0.02925 & 0.003265 & 0.002447 & 0.007718 \\
10 & 0.4579 & $8.833 \mathrm{e}-005$ & $4.663 \mathrm{e}-008$ & 0.0002002 \\
15 & 0.9165 & $2.633 \mathrm{e}-006$ & $7.816 \mathrm{e}-014$ & $5.872 \mathrm{e}-006$ \\
\hline
\end{tabular}


the NP criterion while keeping the probability of false alarm within the desired limits for specified values of SNR of the PU-CR link.

\section{Endnote}

${ }^{a}$ We are skipping analytical details here and writing the final expressions to avoid repetition.

\section{Competing interests}

The authors declare that they have no competing interests.

\section{Acknowledgements}

This work was partially supported by the IDRC Research Grant RP02253.

\section{Author details}

${ }^{1}$ Department of Electronics and Telecommunication Engineering, National Institute of Technology - Raipur, Chhattisgarh 492010, India. ${ }^{2}$ Department of Electrical Engineering, Indian Institute of Technology - Delhi, Hauz Khas, New Delhi 110016, India.

\section{Received: 6 August 2011 Accepted: 22 May 2013}

Published: 14 June 2013

\section{References}

1. S Haykin, Cognitive radio: brain-empowered wireless communications, IEEE J. Sel. Areas Commun. 23, 201-220 (2005)

2. RW Broderson, A Wolisz, D Cabric, SM Mishra, D Willkomm, CORVUS: A Cognitive Radio Approach for Usage of Virtual Unlicensed Spectrum. (Univ. California Berkeley Whitepaper, Berkeley, CA, 2004)

3. A Goldsmith, SA Jafar, I Maric, S Srinivasa, Breaking spectrum gridlock with cognitive radios: An information theoretic perspective,Proc. IEEE. 97(5), 894-914 (2009)

4. Z Quan, S Cui, AH Sayed, Optimal linear cooperation for spectrum sensing in cognitive radio networks, IEEE J. Sel. Topics in Sig. Proc. 2(1), 28-40 (2008)

5. J Unnikrishnan, W Veeravalli, Cooperative sensing for primary detection in cognitive radio, IEEE J. Sel. Topics in Sig. Proc. 2, 18-27 (2008)

6. E Peh, Y-C Liang, in Proceedings of the IEEE Wireless Communications and Networking Conference (WCNC). Optimization for cooperative sensing in cognitive radio networks (March 2007, pp. 27-32. Hong Kong

7. W Zhang, RK Mallik, KB Letaief, Optimization of cooperative spectrum sensing with energy detection in cognitive radio networks, IEEE Trans. Wireless Commun. 8(12), 5761-5766 (2009)

8. Y Liu, C Zeng, H Wang, G Wei, in Proceedings of the 2nd International Conference on Advanced Computer Control (ICACC). Energy detection threshold optimization for cooperative spectrum sensing Shenyang, March 2010), pp. pp.566-570

9. JG Proakis, M Salehi, Digital Communications, 5th edn. (McGraw-Hill, New York, 2008)

10. H Urkowitz, Energy detection of unknown deterministic signals, Proc. IEEE. 55(4), 523-531 (1967)

11. VI Kostylev, in Proceedings of the IEEE Int. Conf. on Communications (ICC'02), Energy detection of a signal with random amplitude (New York, 28 April-2 May 2002)

12. FF Digham, M-S Alouini, MK Simon, On the energy detection of unknown signals over fading channels, IEEE Trans. Commun. 55(1), 3575-3579 (2007)

13. J Ma, G Zhao, Soft combination and detection for cooperative spectrum sensing in cognitive radio networks. IEEE Trans. Wireless Commun. 7(11) 4502-4507 (2008)

14. G Ganesan, Y Li, Cooperative spectrum sensing in cognitive radio, part I: two user networks, IEEE Trans. Wireless Commun. 6(6), pp.2204-2213 (2007)

15. YC Liang, Y Zeng, Peh ECY, AT Hoang, Sensing-throughput tradeoff for cognitive radio networks, IEEE Trans. Wireless Commun. 7(4), pp.1326-1337 (2008)

16. S Atapattu, C Tellambura, $\mathrm{H}$ Jiang, in Proceedings of the IEEE Int. Conf. on Industrial and Information Systems (ICIIS). Energy detection of primary signals over $\eta$ - $\mu$ fading channels (Sri Lanka, December 2009), pp. pp.118-122

17. S Atapattu, C Tellambura, H Jiang, Energy detection based cooperative spectrum sensing in cognitive radio networks, IEEE Trans. Wireless Commun. 10(4), 1232-1241 (2011)

18. SP Herath, N Rajatheva, C Tellambura, Energy detection of unknown signals in fading and diversity reception, IEEE Trans. Commun . 59(9), 2443-2453 (2011)

19. S Atapattu, C Tellambura, H Jiang, Performance of an energy detector overcChannels with both multipath fading and shadowing, IEEE Trans. Wireless Commun. 9(12), 3662-3670 (2010)

20. EH Gismalla, E Alsusa, Performance analysis of the periodogram-based energy detector in fading channels, IEEE Trans. Sig. Proc. 59(8), 3712-3721 (2011)

21. AW Min, X Zhang, KG Shin, Detection of small-scale primary users in cognitive radio networks, IEEE J. Sel. Areas Commun. 29(2), 349-361 (2011)

22. S Atapattu, C Tellambura, H Jiang, in Proceedings of the IEEE Int. Conf. on Communications (ICC'11). Spectrum sensing via energy detector in low SNR (Kyoto, June 2011), pp. 5-9

23. S Atapattu, C Tellambura, H Jiang, in Proceedings of the IEEE Int. Conf. on Industrial and Information Systems (ICIIS). Spectrum sensing in low SNR diversity combining and cooperative communications (Kandy, 16-19 August 2011), pp. 13-17

24. P Jia, M Vu, T Le-Ngoc, SC Hong, V Tarokh, "Detection of small-scale primary users in cognitive radio networks," IEEE J. Sel. Areas Commun 29(2), 276-289 (2011)

25. A Papoulis, SU Pillai, Probability, random variables and stochastic processes, 4th edn. (McGraw-Hill, New York, 2002)

26. M Abramowitz, IA Stegun, Handbook of mathematical functions with formulas, graphs, and mathematical tables, 9th edn. (Dover, New York, 1970)

27. HL Van Trees, Detection, estimation, and modulation theory, (Wiley, New York, 1968). Part 1

28. MR Bhatnagar, A Hjørungnes, M Debbah, Delay-tolerant decode-and-forward based cooperative communication over Ricean channels,IEEE Trans. Wireless Commun. 9(4), 1277-1282 (2010)

29. IEEE P802.22/D0.5, Draft Standard for Wireless Regional Area Networks Part 22: Cognitive Wireless RAN Medium Access Control (MAC) and Physical Layer (PHY) specifications: Policies and procedures for operation in the TV Bands. (IEEE, Piscataway, 2008)

doi:10.1186/1687-1499-2013-165

Cite this article as: Singh et al:: Threshold optimization of a finite samplebased cognitive radio network using energy detector. EURASIP Journal on Wireless Communications and Networking 2013 2013:165.

\section{Submit your manuscript to a SpringerOpen ${ }^{\circ}$ journal and benefit from:}

- Convenient online submission

Rigorous peer review

- Immediate publication on acceptance

- Open access: articles freely available online

- High visibility within the field

Retaining the copyright to your article

Submit your next manuscript at $>$ springeropen.com 